\title{
Direct numerical simulation of deformation twinning in polycrystals
}

\author{
Shawn A. Chester ${ }^{\dagger *}$, Joel V. Bernier ${ }^{\dagger}$, Nathan R. Barton ${ }^{\dagger}$, \\ Levente Balogh ${ }^{\ddagger}$, Bjørn Clausen ${ }^{\diamond}$, and John K. Edmiston ${ }^{\dagger}$ \\ ${ }^{\dagger}$ Computational Engineering Division \\ Lawrence Livermore National Laboratory \\ Livermore, CA 94550, USA \\ * Department of Mechanical Engineering \\ New Jersey Institute of Technology \\ Newark, NJ 07102, USA \\ ${ }^{\ddagger}$ Mechanical and Materials Engineering \\ Queen's University \\ Kingston, ON, Canada \\ ${ }^{\diamond}$ Materials Science and Technology \\ Los Alamos National Laboratory \\ Los Alamos, NM 87545, USA
}

August 19, 2016

\begin{abstract}
The ability to directly simulate the formation of twin domains in crystalline materials is of interest to the mechanics of materials community. While extensive work has been published on homogenized crystal mechanics treatments of twinning, publications that directly capture twin domain formation are relatively rare. This is due both to the complexities of model development and to the computational costs involved. We present results from simulations of twinning in polycrystals with finite elements that spatially resolve twin formation. Effects of interest include the role of stress concentrations in twin initiation, the interactions among twin systems, and competition between deformation twinning and dislocation glide plasticity. We anticipate that results from models that spatially resolve twin formation will help to inform more homogenized multiscale schemes. We show basic features of the model via numerical simulations on a model polycrystal system in simple shear, and also examine the complete model through large scale simulation of a dynamically compressed polycrystal. Comparisons are made between experimental data from far-field high energy diffraction microscopy (HEDM) and numerical simulations for a magnesium alloy polycrystal in compression. We finish with some final remarks and directions for future work.
\end{abstract}

Keywords: Twinning; Crystal Plasticity; High-energy X-ray Diffraction Microscopy; Magnesium Alloy; Tantalum

\footnotetext{
*E-mail address: shawn.a.chester@njit.edu, chester2@llnl.gov
}

(C) 2016. This manuscript version is made available under the Elsevier user license http://www.elsevier.com/open-access/userlicense/1.0/ 


\section{Introduction and background}

Mechanical twinning plays an important role in the plastic deformation of many crystalline materials. Low symmetry crystals in which dislocation mediated slip does not readily accommodate general deformation modes are especially prone to twinning. For example, in hexagonal closepacked (HCP) crystals slip may be restricted to a relatively small number of basal or prismatic slip systems, and there may not be any readily activated crystallographic slip systems that allow deformation along the c-axis. General loading conditions will therefore tend to produce a combination of deformation by twinning and slip. Additionally, even in high symmetry crystals with ample slip systems such as face centered cubic (FCC) and body centered cubic (BCC) crystals, it is well known that low temperatures and/or high strain rates tends to promote deformation twinning [1, 2, 3, 4]. For example, in BCC tantalum, it is postulated that twinning occurs when the strain rate is sufficiently high that the usual dislocation mechanisms do not provide rapid enough relaxation to prevent the stress from building. There is also experimental evidence to suggest a complex interplay between dislocation density evolution and deformation twinning found in BCC tantalum [4], making tantalum a very interesting material to consider. In what follows, our focus will be concentrated on HCP magnesium alloy AZ31, as well as BCC tantalum.

In modeling the plastic deformation of polycrystalline materials several forms of "crystal plasticity" are in use, such as Visco-Plastic Self Consistent (VPSC), Visco-Plastic Fast Fourier Transform (VPFFT), Elastic-VPSC, Elastic-VPFFT, and full Finite Element (FE) based methods [5, 6, 7]. There are also numerous partial differential equation solution methods in addition to typical finite element methods, such as the Fast Fourier Transform (FFT) based schemes [8, 9], and also a wide literature using phase-field methods [10], although much of the phase-field methods have been for phase transformations [11].

Crystal plasticity based models were initially driven by the seminal contributions of Taylor and Schmid $[12,13]$ based on early qualitative observations. Quantitative analysis of slip-based plastic deformation is feasible by a number of constitutive models [14, 15]. While slip-dominated crystal plasticity has been widely studied and generally accepted, many questions still exist for twinning. For example, open questions remain regarding twin initiation and the interactions among twins and obstacles. Such obstacles include other twins, grain boundaries, dislocation networks, and so on. Nevertheless, deformation twinning has been incorporated into models that include the combined effects of twinning and slip as inelastic mechanisms (for example, [16] and references therein for recent examples). A more complete understanding of such interactions should lead to improved constitutive models and predictive simulation capabilities.

In regard to constitutive modeling of twinning within the crystal plasticity context, one of the earlier contributions was made by van Houtte [17] where it was postulated that twinning could be modeled by reorienting the twinning grain. However, it was shown later that numerous grains are required for such an assumption to obtain reasonable results for a polycrystal calculation [18]. Kalindindi [19] proposed a constitutive model in which twinning was modeled in a manner analogous to slip, and dislocation slip was considered in both the parent and child (twinning-produced) grains. Later Staroselsky and Anand [20] developed a constitutive model for HCP metals based on Van Houtte's criteria for reorientation due to twinning in addition to a modified flow rule to account for grain boundary plasticity accommodation. The model was shown to simulate the average stressstrain response as well as texture in magnesium rods and plates. Typically, these types of modeling efforts do not spatially resolve twin dominions, but rather track a homogenized quantity, such as the twin volume fraction at a material point. Additionally, a few other research groups [21, 22, 23] have devised and implemented schemes based on evolving an orientation distribution function including the non-local contributions from mechanical twinning. However, these types of schemes also do not 
spatially resolve individual twin domains.

In addition to finite element based approaches to polycrystal plasticity, the self-consistent approach is also prevalent in the literature, and worth mention here. Self-consistent type approaches have also shown great success in describing the deformation of polycrystals when slip is the dominant mechanism of inelasticity and these methods have been extended to include twinning as well $[24,25]$. The underlying assumption is that each grain may be treated as if it were embedded in a homogeneous effective medium (HEM) which is meant to represent the polycrystal. Then using an Eshelby approach [26] with appropriate analysis the stress-strain response of the grain may be related to the macroscopic response of the HEM. However, means of effectively including the spatially localized nature of twinning in such frameworks is an active area of research [27, 28, 29, 10, 30, 31], and for that reason we use a finite element based approach in this work.

Multiscale modeling approaches have seen increased popularity and utility in recent years, and we have pursued both concurrent and information-passing types of multiscale models in previous work [32, 33, 23, 34, 35, 36]. Our multiscale approach in [36] makes use of an information-passing paradigm: simulation results from lower length-scale calculations are used to define functional forms and parameters at the next larger length-scale. This approach is in contrast with direct (concurrent) multiscale embedding, which is sometimes employed when unit mechanisms are difficult to identify or a stronger coupling between scales is required [32, 33, 23, 34, 35]. Much of our previous modeling work assumed dislocation motion as the dominant mechanism for inelasticity with hardening arising from the interactions among dislocations. In this work, we focus attention to direct numerical simulation of twin domains at the crystal level.

Capturing the correct twin domain structure in a model would enable:

- Examining sub-grain level stress heterogeneity and corresponding effects on twinning.

- Capturing autocatalytic effects - twins running into boundaries producing stress concentrations that cause twinning on the other side of the boundary.

- Capturing intra-grain transitions in microstructure due to twinning.

- Informing more homogenized phenomenological crystal models or macroscopic models.

- Creation of a computational capability that allows for model validation by direct comparison with in situ grain-resolved X-ray diffraction data that resolves twin domains.

- Examining anisotropic effects in twin formation resulting from changes in deformation mode.

Our objective in this work is to report on our simulations that spatially resolve twin domains and to compare our crystal level simulations with experimental data. Additionally, we discuss how our results could effect higher-scale constitutive theories in a multiscale scheme. Indeed, we anticipate that the incorporation of such crystal level effects into a multiscale strength model will provide additional details to better predict material response in real-world applications.

The remainder of the paper is organized as follows. In Section 2 the basics of the crystal level constitutive model are summarized. In Section 3, using tantalum as an example, we provide an overview of the capabilities of the constitutive model. Specifically Section 3.1 provides model details for tantalum, and Section 3.2, via numerical simulation, shows the basic constitutive model behavior for an idealized tantalum system. That is followed by Section 3.3 where we present a large scale simulation of a tantalum polycrystal. As discussed in [4], these results for tantalum capture interesting aspects of experimentally observed behavior. A comparison between high energy diffraction microscopy data and simulations for a magnesium alloy is presented in Section 4. And lastly, we finish with some concluding remarks in Section 5. 


\section{Review of the constitutive model}

The model allows for deformation by thermo-elastic lattice distortion, lattice rotation, dislocation based slip, and twinning [37, 38, 39]. All deformation modes can occur in both the parent and twin domains. In the simulations that follow, BCC tantalum is modeled with twelve $\{1 \overline{1} 0\}\langle 111\rangle$ slip and twelve $\{211\}\langle\overline{1} 11\rangle$ twinning systems. ${ }^{1}$ Further, HCP magnesium alloy AZ31 is modeled with three basal $\{0001\}\langle 1120\rangle$, three prismatic $\{1 \overline{1} 00\}\langle 1120\rangle$, and six pyramidal $\langle c+a\rangle\{11 \overline{2} 2\}\langle\overline{1} \overline{1} 23\rangle$ slip systems, as well as six $\{10 \overline{1} 2\}\langle\overline{1} 011\rangle$ "tensile" twinning systems. More slip systems can be used to model pencil glide type behavior. In most publications related to the modeling framework $[37,38,39]$ the reference rates for the dislocation slip based deformation modes have been fixed. In other crystal level work [40], these rates have been made to depend on the current dislocation density, more like what is done in certain models meant for high rates, for example [41, 42, 43]. Such considerations can influence slip/twinning transitions and relevant studies are ongoing [44]. In what follows we consider these reference rates to depend on the current dislocation density. The model uses a single dislocation density for all slip systems in each constituent (parent or twin variant), and there are standard production and annihilation terms in the dislocation density kinetics. Currently, we assume that twins are allowed to revert to the parent, but may not internally twin, an assumption that reduces the computational cost and complexity. The model uses a Mie-Grüneisen equation of state for the pressure-volume relationship [45].

\subsection{Summary of relevant constitutive equations}

\subsubsection{Kinematics}

Within each constituent (parent or twin), we perform a multiplicative decomposition of the deformation gradient,

$$
\mathbf{F}=\mathbf{V} \cdot \mathbf{R} \cdot \mathbf{F}^{p} .
$$

Lattice rotation is captured by the proper rotation $\mathbf{R}$, while $\mathbf{V}$ is a symmetric tensor that captures the thermo-elastic stretch of the lattice, plastic deformation via slip is captured by $\mathbf{F}^{p}$.

Let constituents be indicated by Greek indicies, with all summations over constituents indicated explicitly. With constituent volume fraction $v_{\zeta}$ and velocity gradient $\mathbf{L}_{\zeta}$ in constituent $\zeta$, the total apparent velocity gradient is given by

$$
\langle\mathbf{L}\rangle=\mathbf{L}^{\chi}+\sum_{\zeta} v_{\zeta} \mathbf{L}_{\zeta}, \quad \mathbf{L}^{\chi}=\sum_{i} \mathbf{L}_{(i)}
$$

with $\mathbf{L}^{\chi}$ due to twinning. Within each constituent $\zeta$, the velocity gradient $\mathbf{L}_{\zeta}$ captures all nontwinning related deformations. When no twinning modes $\left(\mathbf{L}_{(i)}\right)$ are active $\mathbf{L}^{\chi}$ reduces to $\mathbf{0}$, and the apparent velocity gradient reduces to a volume average over the constituents. However, we note that the $\mathbf{L}_{(i)}$ involve the twin shear as well as the stretch of the lattice in going from the parent to the twin orientation under the current local stress state [37, 38, 39].

\subsubsection{Slip and twinning rates}

Within a given constituent (parent or twin variant), the form of the slip kinetics is relatively simple, with each slip system $\alpha$ having a shear rate

$$
\dot{\gamma}^{\alpha}=\left(\frac{1}{\dot{\gamma}_{\mathrm{w}}^{\alpha}}+\frac{1}{\dot{\gamma}_{\mathrm{r}}^{\alpha}}\right)^{-1}
$$

\footnotetext{
${ }^{1}$ In Section 3.2, we limit the material to a single twin system so that we may more clearly understand the behavior of the constitutive model in a simplified setting.
} 
which arises from dislocations spending time both overcoming barriers by thermal activation $\dot{\gamma}_{\mathrm{w}}^{\alpha}$, and gliding $\dot{\gamma}_{\mathrm{r}}^{\alpha}[1,40,46]$. The shear rates depend on the resolved shear stress, $\tau^{\alpha}$, the current dislocation density measure, $h$, and the temperature $\theta$,

$$
\dot{\gamma}_{\mathrm{r}}^{\alpha}=\dot{\gamma}_{\mathrm{r}}^{\alpha}\left(\tau^{\alpha}, h, \theta\right), \quad \dot{\gamma}_{\mathrm{w}}^{\alpha}=\dot{\gamma}_{\mathrm{w}}^{\alpha}\left(\tau^{\alpha}, h, \theta\right),
$$

with specific forms provided later. In numerical implementations, care must be taken over the sign of $\tau^{\alpha}$, but we do not encumber our following discussions with this detail. The strength contribution from dislocation density takes the form

$$
g^{\alpha}=g_{\mathrm{o}}+s \sqrt{h}
$$

Where $h$ is a unitless relative dislocation density

$$
h \equiv \frac{\rho^{\perp}}{\rho_{O}^{\perp}}
$$

with $\rho^{\perp}$ being the actual number density of dislocations and $\rho_{o}^{\perp}$ being a reference value. The classical hardening form in Equation 5 accounts for dislocation interactions and the offset value $g_{\mathrm{o}}$ can be motivated by phenomenology of grain size effects [47, 48].

The relative dislocation density evolves according to

$$
\dot{h}=\left(k_{1} \sqrt{h}-k_{2} h\right) \dot{\gamma} \quad \text { where } \quad k_{2}=\left(k_{2 \mathrm{o}}\right)\left(\frac{\dot{\gamma}_{\mathrm{ko}}}{\dot{\gamma}}\right)^{1 / n}
$$

with an initial value $\left.h\right|_{t=0}$. The first term accounts for generation as moving dislocations drag out new dislocation line length and the second term accounts for dislocation annihilation [49]. Within each constituent, a single average dislocation density is used for all slip systems, and the evolution is driven by the net slip system shearing rate $\dot{\gamma}=\sum_{\alpha}\left|\dot{\gamma}^{\alpha}\right|$. We have assumed a net shearing rate in order to focus attention on the novel aspects related to twinning in this work. As shown in [50], this assumption keeps the hardening model simple but still captures the basic aspects of material response.

Unidirectional twinning kinetics follow a simple power-law form [37, 38], with

$$
\dot{m}_{(i)}=\left\{\begin{array}{cl}
\left(\dot{\Phi}^{\mathrm{o}}\right)\left(\frac{f_{(i)}-\xi_{\mathrm{t}}}{g_{(i)}}\right)^{1 / r} & \text { for } f_{(i)}>\xi_{\mathrm{t}(i)} \\
0 & \text { for } f_{(i)} \leq \xi_{\mathrm{t}(i)}
\end{array} .\right.
$$

Where $f_{(i)}$ is the driving force and $\xi_{\mathrm{t}}$ a threshold. The resistance to twinning, like the slip system strength, is obtained from the dislocation density. It is assumed to obey the relation

$$
g_{(i)}=\xi_{\mathrm{o}}+\xi \sqrt{h_{\eta_{\mathrm{fr}}(i)}} .
$$

We use the dislocation density in the twinning constituent, $\eta_{\mathrm{fr}}(i)$, because the domain boundary is sweeping this volume as twinning occurs, which has a value $h_{\eta_{\mathrm{fr}}(i)}$ based on prior history. To keep the constitutive forms simple, we assume that the dislocation density of the parent is transferred to the twin without modification. However, we note that other more complex assumptions are being explored in the literature [51,52]. The coefficient $\xi$ is much like $s$ in Equation 5 in that it controls the degree to which dislocations serve as obstacles to deformation. Prior deformation of a material often increases the driving force needed for transformation (for example [53]) and $\xi$ modulates the influence of dislocation density in producing this phenomenon. 


\subsubsection{Interaction energy}

The interaction energy model penalizes the coexistence of parent and twin domains. Many examples may be found in the literature, for example [54] is a simple example in the case for a system with only two constituents. Additionally, [55] has the quadratic term, as well as an additional quadratic form to handle gradient terms to make the model nonlocal. In general readers are referred to [56] for background on these forms.

The energy is approximated by the form

$$
E^{\mathrm{r}}=\sum_{\zeta, \nu=1}^{n_{\mathrm{c}}} m_{\zeta} A_{\zeta \nu}^{\mathrm{r}} m_{\nu}
$$

where $n_{c}$ is the number of constituents (in this case the number of twins plus one parent), and

$$
A_{\zeta \nu}^{\mathrm{r}}=\left[\begin{array}{cc}
0 & C^{\mathrm{r}_{\mathrm{b}}} \cdots C^{\mathrm{r}_{\mathrm{b}}} \\
C^{\mathrm{r}_{\mathrm{b}}} & \\
\vdots & C^{\mathrm{r}_{\mathrm{a}}}\left(1-\left|\mathbf{n}_{\zeta} \cdot \mathbf{n}_{\nu}\right|\right) \\
C^{\mathrm{r}_{\mathrm{b}}} &
\end{array}\right]
$$

so that $C^{r_{a}}$ penalizes coexistence of twins (according to the degree to which their nominal habit planes $\mathbf{n}_{\zeta}$ intersect) and $C^{\mathrm{r}_{\mathrm{b}}}$ penalizes coexistence of twins with the parent crystal. Driving force contributions are determined from the partial derivatives of the energy with respect to mass fraction

$$
f_{(i)}^{\mathrm{r}}=-\frac{\bar{\rho}_{\eta_{\mathrm{fr}}(i)}}{\bar{\rho}}\left(\frac{\partial E^{\mathrm{r}}}{\partial m_{\eta_{\mathrm{to}}(i)}}-\frac{\partial E^{\mathrm{r}}}{\partial m_{\eta_{\mathrm{fr}}(i)}}\right) .
$$

With $\bar{\rho}$ denoting the referential mass density, the leading term, $\bar{\rho}_{\eta_{\mathrm{fr}}(i)} / \bar{\rho}$, provides a scaling between reference volumes. We note that the parameters $C^{\mathrm{r}_{\mathrm{a}}}$ and $C^{\mathrm{r}_{\mathrm{b}}}$ in the interaction energy can cause convergence issues when using implicit numerical schemes. When the penalty for variant coexistence is high, the system may become numerically unstable in the absence of regularization from inertial effects, however this behavior is consistent with "pop-in" events in which twins form abruptly.

\section{Behavior and capabilities of the constitutive model}

In this section we present the results of numerical simulations to show typical results and the model behavior. These simulations illustrate effects that are captured by resolving twin domain formation, such as intra-granular microstructural changes and autocatalytic effects. Specifically, we consider an idealized two dimensional tantalum polycrystal in simple shear (Section 3.2) and a fully three dimensional polycrystal under uniaxial compression (Section 3.3).

\subsection{Constitutive model details for tantalum}

Aspects of the model for tantalum are similar to those reported in [3], although in that previous work there was no attempt to spatially resolve twin domains and there are some corresponding differences in parameters. Recalling Equation 4, the shear rates depend on the resolved shear stress, $\tau^{\alpha}$, the current dislocation density measure, $h$, and the temperature $\theta$. For the glide of dislocations between sets of obstacles, phonon drag is assumed to be the dominant rate-limiting mechanism with the form

$$
\dot{\gamma}_{\mathrm{r}}^{\alpha}=\left(\dot{\gamma}_{\mathrm{ro}} h\right)\left(1-\exp \left(-\frac{\tau^{\alpha}}{D_{\mathrm{r}}}\right)\right)
$$


accounting for saturation as the dislocation velocity approaches the shear wave speed [43, 1] (note that in other work a Lorentz factor is used instead of the exponential form shown here [41], but the two forms are substantially equivalent in the range of dislocation velocities probed in this work). The prefactor $\left(\dot{\gamma}_{\text {ro }} h\right)$ is motivated by the idea is that the total shearing rate is proportional to the number density of dislocations participating in the slip process, especially at high strain rates. This form does not directly account for acceleration effects [57] and, as in [41], assumes that the drag stress $D_{\mathrm{r}}$ is independent of temperature over the range of interest. For the thermally activated portion of the model, the balanced form

$$
\left.\dot{\gamma}_{\mathrm{w}}^{\alpha}=\left(\dot{\gamma}_{\mathrm{wo}} h\right)\left(\exp \left(-\frac{\Delta G^{\alpha}\left(\tau^{\alpha}\right)}{\kappa \theta}\right)-\exp \left(-\frac{\Delta G^{\alpha}\left(-\tau^{\alpha}\right)}{\kappa \theta}\right)\right)\right)
$$

gives reasonable behavior up to high temperatures $[58,38,59]$. Dependence on the resolved shear stress is given by

$$
\Delta G^{\alpha}\left(\tau^{\alpha}\right)=g_{\circ} b^{3} \mu^{\alpha}\left[1-\left(\frac{\tau^{\alpha}-g^{\alpha}}{\tau_{\mathrm{p}}}\right)\right] \quad \text { for } \quad \tau^{\alpha} \leq g^{\alpha}+\tau_{\mathrm{p}}
$$

where $\mu^{\alpha}$ is the shear modulus resolved onto the slip system, and $b$ the magnitude of the Burgers vector, and $\tau_{\mathrm{p}}$ the Peierls stress. There are several simplifications to the thermally activated contribution that should be made clear. First, we consider the Peierls stress $\tau_{\mathrm{p}}$ the same for all slip systems, and second we assume the typical exponents " $p$ " and " $q$ " (cf. [60] and Equation 3 in [3]) are both unity. Additional details of the numerical implementation of the slip kinetics may be found in [40].

Various coefficients in the above equations take on specific meanings based on the underlying mechanical assumptions, and those are described here. For $s$ in Equation 5,

$$
s=\beta b G_{0} \sqrt{\rho_{o}^{\perp}}
$$

with $\beta$ being a parameter of order unity and $G_{0}$ being the reference shear modulus.

For $\dot{\gamma}_{\text {wo }}$ in Equation 14,

$$
\dot{\gamma}_{\text {wo }}=\eta_{\mathrm{p}} b^{2} \rho_{o}^{\perp} \nu
$$

with $\nu$ being the attempt frequency factor $([61,1])$ and $\eta_{\mathrm{p}}$ being a microstructure factor of order unity. The thermally activated barrier under consideration is the Peierls barrier and we consider all dislocations as participating, instead of assuming a separate population of mobile dislocations.

And finally for $\dot{\gamma}_{\text {ro }}$ in Equation 13,

$$
\dot{\gamma}_{\text {ro }}=\eta_{\mathrm{p}} b \rho_{o}^{\perp} c_{0}
$$

with $c_{0}$ being the reference shear wave speed $\left(c_{0}=\sqrt{G_{0} / \bar{\rho}}\right.$ in which $\bar{\rho}$ is the reference mass density not the reference dislocation density). This is chosen to be consistent with the dislocation velocity approaching $c_{0}$ for large $\tau^{\alpha} / D_{\mathrm{r}}$.

The full set of material parameters used for tantalum in the simulations is provided in Table 1.

\subsection{Simple shear of an idealized polycrystal}

We consider a planar geometry consisting of 37 hexagonal grains surrounded by a homogeneous embedding medium. Figure 1 shows a schematic of the geometry. Further, we idealize the material such that only one twin system is available, and the twin shear directions are shown by the thick lines in each grain in Figure 1. Each of the idealized grains are 20 microns across and the total 
geometry is 220 microns square. Lastly, twinning is most typically observed in tantalum at either low temperatures, or high strain rates. In the simulations that follow we will use relatively high strain rates $\left(\sim 10^{5} /\right.$ second and up) to observe twins and not just slip.

The mesh used in the finite element simulation of the Ta polycrystal consists of 114400 elements with 230202 nodes total (we use a single element through the thickness). The mesh of each grain, as shown in Figure 2 consists of 400 reduced integration elements (an approximate element size of 1 micron), with 84800 reduced integration elements for the elastic medium. The finite element program ale3d was used for our simulations [62]. ale3d is a sophisticated finite element program, and it allows the simulation to switch from an implicit quasi-static solution method to a dynamic explicit solution method at the onset of twinning when using reduced integration elements. As briefly mentioned earlier, switching to a dynamic explicit solution procedure at the onset of twinning is important when a "pop-in" event is expected and inertial effects serve to regularize the problem.

To impart the macroscopic simple shear deformation, all nodes along the outer edge of the homogeneous elastic medium are prescribed appropriate displacement boundary conditions such that $\mathbf{F}=\mathbf{1}+\gamma \mathbf{e}_{1} \otimes \mathbf{e}_{2}$. The simulations were carried out to a macroscopic shear strain of $\gamma=0.15$. As mentioned previously, material parameters used for the tantalum in the simulations are provided in Table 1. For the homogeneous embedding medium, we use an elastic material model with a shear modulus of $69 \mathrm{GPa}$ and Poisson ratio of 0.34 , these parameters approximate generic isotropic tantalum.

Figure 3 and Figure 4 show a few snapshots of the twin mass fraction in the idealized tantalum polycrystal at various levels of macroscopic deformation at fixed shear strain rates of $10^{5} /$ second, and $10^{6}$ /second, respectively. Upon comparison between the two figures, the most striking result is the higher degree of twinning in the faster strain rate. An increase in twin formation with strain rate over this range is consistent with experimental results [3].

An interesting result stemming from the direct simulation of spatially resolved twin domains is the interaction of twins across grain boundaries. For example, as a twin from grain "A" impinges upon a grain boundary, if the orientations are suitably favorable, the local stress field may be intense enough to form a twin in grain "B." This sort of behavior is roughly detected in the snapshots shown in Figures 3 and 4.

A feature of interest is the apparent pairs of twins that form during the deformation. In experimental micrographs, for a given twin system one typically observes bands of twins aligned with the nominal crystallographic twin plane. However, in these simulations pairs of twins form nearly perpendicular to each other. They are most readily seen in the early stages of deformation in Figures 3 and 4. A simple analysis provided in the appendix shows that this is an artifact of the model, and a topic for future work. For the sake of completeness, an alternative analysis originally proposed by Orowan [63], and reviewed in [2] attributes surface energy as the main factor driving the selection of habit plane, in the sense that one surface energy is lower than the other. However, we leave a detailed study of this for future work as this is still an active area of research.

Lastly, it is worth noting that the mass fractions tend to form twin domains in a roughly planar fashion, as is expected for twins and consistent with the idealized compatibility type considerations in the appendix. However, there is nothing in the model that expressly requires planar twins - it arises naturally as a result of the mechanics involved. We note that the interaction energy coefficients $\left(C^{\mathrm{r}_{\mathrm{a}}}\right.$ and $C^{\mathrm{r}_{\mathrm{b}}}$ in Equation 11) have to be set suitably high to get distinct twin formation. If those values are low, then more diffuse twin mass fractions are observed and the model is effectively resolving the average twin mass fraction over a larger volume of material. Also, given that there is 
just one twin system here, the form of Equation 11 is particularly simple and reduces to

$$
A_{\zeta \nu}^{\mathrm{r}}=\left[\begin{array}{cc}
0 & C^{\mathrm{r}_{\mathrm{b}}} \\
C^{\mathrm{r}_{\mathrm{b}}} & 0
\end{array}\right]
$$

so that indeed no habit plane assumptions are built into Equation 11 for the results in this section.

\subsection{Uniaxial compression of a tantalum polycrystal}

Large-scale computing resources are used to simulate the twin formation during the deformation of a three dimensional polycrystal of tantalum. The same set of material parameters are used here as in the previous tantalum simulation in simple shear, but with the full set of twin systems available. These simulations are also used to investigate the interaction of twin growth with dislocation density - a complex topic of ongoing interest [3,4]. The computational expense of the constitutive model combined with the mesh resolution requirements to capture effects in a polycrystal make these simulations computationally demanding.

The initial domain was $60 \times 60 \times 60$ microns, containing 512 grains, with a typical grain size of 10 microns. All aspects of the computation, including grain structure, are periodic laterally so the there is effectively no lateral boundary. For the simulation, a mesh of $384 \times 384 \times 384=56,623,104$ elements in total was used, leading to roughly 110600 elements per grain on average. A depiction of the initial microstructure is provided in Figure 5, where the element boundaries are not shown for clarity in the microstructure.

The polycrystal is deformed in uniaxial compression with periodic lateral boundary conditions, so that the pressure increases continuously during deformation, to an axial true strain of 0.2 at a constant axial rate of $10^{7} /$ second. To avoid initial ringing in the solution, the velocity along the compression direction is initialized using a uniform gradient so that the material is undergoing a uniform rate of deformation at the start of the calculation. There are no shocks in this simulation, however this is the typical of a strain rate encountered under extreme loading conditions such as flyer plate impact and laser-driven experiments [1]. The simulation finished in 2268 time steps, taking 5.6 hours of compute time on 32768 processors (2048 nodes) on the Cielo supercomputer.

These simulations are large enough to be relevant to macroscopic material response, but they also provide significant details at the sub-grain scale that are not directly observable in high-rate experiments. Results from early in a calculation are shown in Figure 6. In this calculation, the initial relative dislocation density is taken to be unity, which corresponds to an actual dislocation density appropriate for annealed tantalum. After an initial transient during which the axial deviatoric stress increases rapidly there is a rapid onset of twin formation followed by a growth phase in which the twin mass fraction increases more slowly. The rapid onset of twinning is coincident with a drop in the axial deviatoric stress, as show in Figure 7. Similar rapid initial increase in twin fractions have been suggested from experimental observations [64], albeit through different quantities. The rapid initial twin fraction increase and the drop in axial deviatoric stress could both be associated with twin "pop-in" type events.

Plots over the simulation domain are included at four strain levels in Figure 6. These plots are constructed to help elucidate the nature of the twin evolution. Each plot includes iso-surfaces of twin mass fraction at the current strain and from a previous time 0.005 lower in strain (with the data from the previous time mapped into the current configuration of the body). Current-time iso-surfaces are in blue and previous-time iso-surfaces are in yellow. In each plot, iso-surface levels are at $25 \%$ of the peak twin mass fraction achieved at that time $(0.0178,0.0704,0.15$, and 0.25 respectively for the four plots). 
Several features of the response are observable in Figure 6. Early in the simulation (axial strain of 0.015), many twin domains form with relatively low peak mass fraction. Under mesh refinement, the distribution is similar and the peak local twin mass fractions are higher at a given strain because the finer mesh is better able to capture localized twinning for given interaction energy parameter values. Mesh refinement indicates that the overall stress response and general features of the twin distribution are well-converged. While the interaction energy model does favor abrupt initial formation of twins, it does not have the character of a nucleation model and we are effectively assuming that the twin formation is not nucleation limited. The combined effects of stress variations and other microstructural heterogeneities are assumed to produce ample opportunities for twins to grow if conditions treated by the continuum model are favorable for growth.

As shown in Figure 6, as the twinning progresses a subset of the twins are favored and become dominant. Continued twinning tends to proceed from these existing dominant twins rather than by growth of new twins. Note that the dislocation density is increasing rapidly with strain, and the increased density is modeled as impeding further twinning (Equation 9). In fact if the initial dislocation density is set 100 times higher than the annealed value then no twins are formed at all during the course of the deformation, consistent with experimental results [3, 4]. The factor of 100 is chosen to correspond roughly to an expected increase due to a modest amount of cold working of the material. For the case with lower initial dislocation density the twin fraction continues to grow even after the dislocation density (averaged over the domain) has reached more than 100 times its initial value - see Figure 7. It appears that the local stress concentrations created in the vicinity of existing twins facilitate continued growth once the twins have formed. While the model being employed uses a simple resolved stress condition for twin growth locally, the application of the model to polycrystals deforming heterogeneously provides interesting results about the nature of twin formation. These results about heterogeneity at the scale of twins as they form are in line with challenges faced when attempting to deduce rules for twinning from macroscopic experimental observations. Grain-resolved X-ray diffraction data have also indicated the likely influence of subgrain stress heterogeneities [65]. While we do not know that the functional forms used for the twinning kinetics are correct, it is nevertheless interesting that simple assumptions about the kinetic forms and interactions can produce complex results, albeit through the use of the sizable computing resources mentioned above.

\section{Comparison of the model to an in situ 3-dimensional synchrotron $\mathrm{x}$-ray diffraction experiment on magnesium alloy AZ31}

\subsection{Experimental aspects}

The purpose of this section is to compare the results of a High Energy Diffraction Microscopy (HEDM) experiment in which twins were nucleated in situ with simulations that directly simulate spatially resolved twin domains as described in Section 2. To the best of the authors' knowledge, such a comparison between simulations and experiments is rare with only a few found in the literature [28].

\subsubsection{Methods}

Far-field HEDM measurements were performed on a polycrystalline specimen of magnesium AZ31 subject to uniaxial compression in situ at the Advanced Photon Source beamline 1-ID. The HEDM technique - which includes both near-field [66] and far-field [67] modalities - allows for the characterization of grain-scale microstructure and micromechanical state in polycrystalline materials 
$[68,69]$, including the nucleation and growth of deformation twins [65, 70, 71, 72]. Far-field HEDM [67] - analogous to 3DXRD [73] - provides intergranular orientations, centers of mass, and elastic strain tensors (fully 3-D) for up to on the order of $10^{3}$ grains embedded in a polycrystalline aggregate simultaneously, which makes it particularly well-suited for in situ processing experiments.

Information regarding the sample pedigree can be found in [74]; for clarity, some specific details are repeated here. The source for the compression specimens was a $25.4 \mathrm{~mm}$ thick hot-rolled plate in the soft annealed condition (O temper) with average grain size of $\sim 50 \mu \mathrm{m}$. The rolling texture of the plate had basal poles predominantly oriented perpendicular to the rolling plane. Cylindrical compression samples of $1.2 \mathrm{~mm}$ diameter and $1.8 \mathrm{~mm}$ length were cut by electric discharge machining such that the cylinder axis coincided with the transverse direction of the plate; hence most grains had their $c$-axes roughly perpendicular to the compression direction, which is a favorable orientation to produce $\{10 \overline{1} 2\}\langle\overline{1} 011\rangle$ "tensile" twins under the influence of the Poisson extension. Prior to loading, the grains were grown to an average size of $\sim 200 \mu \mathrm{m}$ via a one hour heat treatment at $500{ }^{\circ} \mathrm{C}$. Strain-free lattice parameters were estimated from the unloaded sample to be $a=3.193(5) \AA, c=5.187(9) \AA$. The relatively coarse-grained micorstructure was chosen to expedite the data reduction.

The rotation series for the HEDM data were taken continuously at a rate of $1^{\circ} s^{-1}$ over a series of prescribed loads. The incident beam was defined by a set of tungsten slits to be $1.5 \times 0.2 \mathrm{~mm}(h \times w)$, which fully illuminated a central slab of the compression sample to mitigate the influence of end effects. The vertical supports of the loadframe restricted the rotation range to $\omega \in\left\{-60^{\circ}, 60^{\circ}\right\}$, and the hydraulic cables on the MTS loadframe precluded symmetric scans on both sides of the sample as is generally preferred for the highest strain resolution [73, 67]. A GE model 41RT flat-panel detector [75] was used to collect the diffraction patterns integrated over each $\Delta \omega$ step. It was roughly centered on and perpendicular to the direct x-ray beam at a distance of $\sim 1800 \mathrm{~mm}$ downstream of the sample. The x-ray wavelength was $0.13695 \AA$, which, with the detector configuration, provided a maximum reciprocal space resolution of $0.94 \AA$. In order to minimize the time required - and sample creep - for a full scan at a given applied load, the rotation step size was chosen to be $\Delta \omega=0.5^{\circ}$. This corresponded to 240 images per load state, which was also the buffer limit for the detector when running in a continuous acquisition mode.

The reference state was measured at an applied stress of $17 \mathrm{MPa}$ to minimize both strain and subsequent movement of the gage volume due to establishing firm contact with the compression platens. Orientation indexing was performed using the novel mapSearch algorithm [76] on the $\{01 \overline{1} 0\},\{0002\},\{01 \overline{1} 3\},\{02 \overline{2} 0\}$, and $\{0004\}$ discrete pole figures, which yielded 33 distinct grains in the diffraction volume. The orientations, centroids, and strains were subsequently refined using all available reciprocal lattice vectors out to the maximum reciprocal space resolution $(\sim 67$ reflections per grain on average). Figure 8 shows the measured $\{01 \overline{1} 2\}$ and $\{0004\}$ pole figures with intensities on a $\log$ scale to enhance the visibility of weak signals. Note that the integrated intensity of each peak on the pole figures scales with the volume of the originating grain. Pole figures calculated from the initial state are also shown in Figure 8, where each reflection was approximated by a gaussian peak in the azimuthal $(\eta)$ and rotation $(\omega)$ directions. A standard deviation of $1^{\circ}$ was employed for visibility, and the intensities are scaled with the volume of the voronoi cell representation of each associated grain. It is noted that the initial grain volumes in the simulation are likely much more uniformly distributed than those in the measurement, in addition to being artificially broadened in the initial state. 


\subsubsection{Observations}

Twinning was observed interactively for multiple grains at an applied compressive stress of $55 \mathrm{MPa}$. Note that due to the quasi-static nature of the measurements, it is not possible to capture individual nucleation events; rather, they are bracketed by the applied load states before and after twins are detected. Previous studies at beamline 1-ID have suggested that the minimum detectable twin size with the instrument as configured is $\sim 1 \mu \mathrm{m}^{3}$. The measured $\{11 \overline{2} 0\}$ and $\{0004\}$ pole figures at $55 \mathrm{MPa}$ are shown in the top row of Figure 9. The diffraction signals from the twins are clearly visible near the equator of the $\{11 \overline{2} 0\}$ pole figure (compare to Figure 8). Twins are detected and associated with parent grains by directly searching the raw image data for diffracted beams in regions calculated for each of the 6 distinct variants in the $\{10 \overline{1} 2\}\langle\overline{1} 011\rangle$ twin system using the parent orientation and center-of-mass coordinates. The detection criterion is a completeness threshold of $\geq 50 \%$ for the same reciprocal lattice vector set used for the initial orientation indexing; a higher threshold could possibly miss small twins for which the weaker structure factor reflections would have intensities below the background level of the detector, while a lower threshold could generate false positives. For convenience, twin variants $\{1,2,3\}$ and $\{4,5,6\}$ represent the pairs of "crossed" $\{10 \overline{1} 2\}$ planes (that is, normals coplanar with and bisected by a $\langle 10 \overline{1} 0\rangle$ direction). Variants 2 and 5 are depicted in Figure 10, which illustrates both the relative orientations of the parent and twin lattices as well as the specific habit plane. The significance of the "crossed" twin pairs is that they share the same resolved shear stress under applied uniaxial loading transverse to the crystallographic $c$-axis (i.e. $\{000 l\}$ ).

In total, 17 of 33 grains are observed to form twins. Of those parent grains, 8 have two or more twins where 6 have the $(2,5)$ crossed twin pair. Two of those 6 have 3 variants: 2,5,6 in one case and 1,2,5 in the other. The other 2 grains have non-crossed twin variant pairs. It is noted that none of the other crossed variant pairs are observed. In the simulation, 21 of 33 grains are observed to form twins after $1 \%$ applied compression. Of those, 11 have 2 variants, all of which are $(2,5)$ pairs. No simulated grains are observed to form more than 2 variants. In addition to the pole figures in Figure 9, further discussion is presented in Section 4.3 where comparison between the experiment and model is performed.

Due to the initial texture of the specimen, there is a clustering of grains around the orientation that has the maximum resolved shear on the $(2,5)$ variant pair. While the local intergranular stresses do deviate from the applied stress state in both the experiment and simulation, the magnitude of the deviation is not large enough to significantly alter the variant selection.

\subsection{Constitutive model details for magnesium AZ31}

Recalling Equation 4, the shear rates depend on the resolved shear stress, $\tau^{\alpha}$, the current dislocation density measure, $h$, and the temperature $\theta$. Unlike the previous example, here we consider power law kinetics for simplicity. Here, for thermally activated slip we take the form

$$
\dot{\gamma}_{\mathrm{w}}^{\alpha}=\frac{\dot{\gamma}_{\mathrm{wo}}}{\sqrt{h}}\left|\frac{\tau^{\alpha}}{g^{\alpha}}\right|^{1 / n}
$$

where, as before, $h$ is a normalized dislocation density, $\tau^{\alpha}$ the resolved shear stress, $g^{\alpha}$ the strength, and $\dot{\gamma}_{\text {wo }}$ a reference shear rate. Also, for gliding we take the form

$$
\dot{\gamma}_{\mathrm{r}}^{\alpha}=\dot{\gamma}_{\mathrm{ro}}\left(1-\exp \left(-\frac{\tau^{\alpha}}{D_{\mathrm{r}}}\right)\right)
$$

with $\dot{\gamma}_{\text {ro }}$ and $D_{\text {r }}$ constants. The form of the strength follows Equation 5, and it's evolution follows Equation 7 . However, here we note that $1 / n$ is set to zero so that $k_{2}$ in Equation 7 is constant 
for Mg AZ31. Similar constitutive forms have been used in the literature to examine twinning in magnesium at the crystal scale [77]. The full set of material parameters used for Mg AZ31 in the simulations is provided in Table 2 .

\subsection{Simulation results and comparison to the experiment}

The specimen used in the numerical simulations contains roughly 120 grains, see Figure 11, with a height of $1 \mathrm{~mm}$ to simulate only the central region of the sample. Given the relatively poor intensity resolution of the detector in this configuration, the initial microstructure for the simulation, shown in Figure 11, was approximated using a Voroni tessellation of the grain centroids alone using far-field data. Each grain was allowed a full set of slip systems and twin systems for inelastic deformation as described earlier. For the numerical simulation, while we model most of the specimen, we analyze results only from the very center when comparing with the experiment to mitigate edge effects. The full mesh consists of 842400 8-noded brick elements and 863871 nodes. As noted previously, we only compare data between the simulation and experiment in the central region of the specimen - that central region is highlighted by the meshed region in Figure 11.

The simulation consists of a single compression step where both the top and bottom faces are displaced quasi-statically to impose a macroscopic state of compression. This is performed at a constant engineering strain rate. In the experiment, the loading was applied in a quasi-static, step-wise manner, with long hold times between increments to allow for the data acquisition in the measurement - not at a constant loading rate. Therefore, quasi-static conditions were assumed for the simulation solution procedure, with constraints applied to eliminate rigid body modes of deformation. Nodes on the top and bottom faces are presumed to be unconstrained by frictional effects, and accordingly lateral movement is allowed on these faces in the simulation.

The material parameters for this simulation were obtained by performing a numerical optimization. Using a Taylor approximation for 625 grains at a material point, stress-strain data as well as pole figure data from [64] were fit using a non-linear least squares method. Typically the stressstrain curve is fit alone without consideration to the texture, however our optimization considers them both in an effort to make sure that the overall twin fraction evolution is captured.

Figure 12a shows a snapshot of the twin mass fractions after roughly $1 \%$ of axial compressive strain. The plot was generated using a non-linear function that highlights twin formation by mapping the mass fractions of the twin variants into red, green, blue, cyan, magenta, and yellow. It is worth noting the prominence of the green and magenta variants. As noted in Section 4.1.2 these are twin systems that are "opposed" across the c-axis as shown in Figure 10, while other variants show up mostly around grain boundaries where there is more stress heterogeneity. Increasing the interaction energy coefficient in the simulation would result in sharpening of the predicted twin domains and could produce autocatalytic effects of the sort discussed in Section 3.

The plot in Figure 12 is sectioned to show the degree of twinning interior to the body and grains. Material interior to the body, particularly material away from the traction-free lateral boundaries, may have a tendency to twin over a wider extent instead of having twin mass fraction concentrations near the grain boundaries. Lastly, the quantitative comparison to the HEDM experimental data is presented as a histogram in Figure 12b. In comparing the experimental observations with the simulation, 14 of the grains behave the same in terms of the specific twin variants formed. An additional 7 grains (in both the experiment and simulation) predict twins, but the variants differ. The remaining 17 grains disagree regarding the nucleation of twins. Note that the numbering of variants is arbitrary, but the orientation conventions are consistent between the simulation and experimental data reduction. Overall, it is of some interest that both the experiments and the model tend to predict more than one twin variant forming in a given parent grain, in contrast with 
some previously used model simplifications $[18,78,79]$. Differences in behavior of grains that form single versus multiple twin variants are of ongoing interest [80].

Differences between the simulation and experiment could have a variety of sources. Further refinement of the model details and parameters might improve the agreement, but are probably not warranted given the other potential sources of differences. The approximate Voroni type microstructure almost certainly misses important aspects of the microstructure and the attendant grain interactions and details in the stress heterogeneity. Increasing the interaction energy to capture sharper twin domains (with corresponding refinement in the finite element mesh) might produce stress concentrations that promote other twin variants. However, one would probably first want to address the tendency of the model to form (see the appendix) both habit plane solutions.

\section{Concluding remarks}

In this paper we have reviewed and illustrated the capabilities of recent efforts for the direct numerical simulation of deformation twin formation in metallic materials. This study provides a basis for further understanding of how twins interact with each other, with grain boundaries, and with dislocation structure. Further, this work helps to provide direction in multiscale modeling efforts.

Indeed, various studies $([2,81])$ have examined the idea of a macroscopic critical resolved shear stress for twinning. Our results, particularly those in Section 3.3, are in line with challenges that researchers have encountered in developing these ideas. Even for the simple crystal model used in this work, the twins may continue to grow by virtue of the local stress fields at the twin boundaries, which can be rather different from grain averaged stresses. This stress heterogeneity is consistent with HEDM experiments such as [65], and may eventually be able to shed light on experimental results [81].

However, significant work remains to be done. Future work could include anisotropic twin mobility, a gradient-informed interaction energy model, and more nucleation-like kinetics. Some of these advances might require non-local information or solution of the twin mass fractions as field quantities.

\section{Acknowledgments}

Part of this work was performed under the auspices of the U.S. Department of Energy by Lawrence Livermore National Laboratory under Contract DE-AC52-07NA27344 (LLNL-JRNL-688817). Capabilities for high energy diffraction microscopy were developed in part under Laboratory Directed Research and Development funding (10-ERD-053, 13-ERD-078). This research used resources of the Advanced Photon Source, a U.S. Department of Energy (DOE) Office of Science User Facility operated for the DOE Office of Science by Argonne National Laboratory under Contract No. DEAC02-06CH11357. The authors are grateful to Ulrich Lienert and Jon Almer of beamline 1-ID at the APS, without whom the measurements would not have been possible. The authors are grateful for the computing resources on the ASC Cielo machine at Los Alamos granted as part of the Capability Computing Campaign (CCC3) - these resources enabled the simulation results shown in Section 3.3.

\section{References}

[1] Marc A. Meyers. Dynamic Behavior of Materials. John Wiley \& Sons, Inc., 1994. 
[2] J. W. Christian and S. Mahajan. Deformation twinning. Progr. Mater. Sci., 39(1-2):1-157, 1995.

[3] Jeffery N. Florando, Nathan R. Barton, Bassem S. El-Dasher, James McNaney, and Mukul Kumar. Analysis of deformation twinning in tantalum single crystals under shock loading conditions. J. Appl. Phys., 113:083522, 2013.

[4] Jeffrey N. Florando, Bassem S. El-Dasher, Changqiang Chen, Damian C. Swift, Nathan R. Barton, James M. McNaney, Kalit T. Ramesh, Kevin J. Hemker, and Mukul Kumar. Effect of strain rate and dislocation density on the twinning behavior in tantalum. AIP Advances, $6(4), 2016$.

[5] F. Roters, P. Eisenlohr, L. Hantcherli, D.D. Tjahjanto, T.R. Bieler, and D. Raabe. Overview of constitutive laws, kinematics, homogenization and multiscale methods in crystal plasticity finite-element modeling: Theory, experiments, applications. Acta Materialia, 58(4):1152 1211, 2010.

[6] H Wang, PD Wu, CN Tomé, and Y Huang. A finite strain elastic-viscoplastic self-consistent model for polycrystalline materials. Journal of the Mechanics and Physics of Solids, 58(4):594$612,2010$.

[7] Ricardo A Lebensohn, Anand K Kanjarla, and Philip Eisenlohr. An elasto-viscoplastic formulation based on fast fourier transforms for the prediction of micromechanical fields in polycrystalline materials. International Journal of Plasticity, 32:59-69, 2012.

[8] R. A. Lebensohn. N-site modeling of a 3d viscoplastic polycrystal using fast Fourier transform. Acta Mater., 49:2723-2737, 2001.

[9] P. Eisenlohr, M. Diehl, R. A. Lebensohn, and F. Roters. A spectral method solution to crystal elasto-viscoplasticity at finite strains. Int. J. Plasticity, 46:37-53, 2013.

[10] J D Clayton and J Knap. Phase field modeling of twinning in indentation of transparent crystals. Modelling Simul. Mater. Sci. Eng., 19(8):085005, 2011.

[11] W. Zhang, J. M. Jin, and A. G. Khachaturyan. Phase field microelasticity modeling of heterogeneous nucleation and growth in martensitic alloys. Acta Mater., 55(2):565-574, 2007.

[12] G.I. Taylor and C.F. Elam. Bakerian lecture the distortion of an aluminum crystal during a tensile test. Proceedings of the Royal Society of London. Series A., 102:643-667, 1923.

[13] E. Schmid and W. Boas. Plasticity of Crystals. Chapman and Hill, London, 1935.

[14] R.J. Asaro and A. Needleman. Overview no. 42 texture development and strain hardening in rate dependent polycrystals. Acta Metallurgica, 33:923-953, 1985.

[15] U. F. Kocks, C. N. Tomé, and H.-R. Wenk, editors. Texture and anisotropy: preferred orientations in polycrystals and their effect on materials properties. Cambridge University Press, 1998.

[16] Yingrui Chang and Dennis M Kochmann. A variational constitutive model for slip-twinning interactions in hcp metals: application to single-and polycrystalline magnesium. International Journal of Plasticity, 73:39-61, 2015. 
[17] P. van Houtte. Simulation of the rolling and shear texture of brass by the taylor theory adapted for mechanical twinning. Acta Metallurgica, 26:591-604, 1978.

[18] C.N. Tome, R.A. Lebensohn, and U.F. Kocks. A model for texture development dominated by deformation twinning: application to zirconium alloys. Acta Metallurgica et Materiallia, 39:2667-2680, 1991.

[19] S. Kalidindi. Incorporation of deformation twinning in crystal plasticity models. Journal of the Mechanics and Physics of Solids, 46:267-290, 1998.

[20] A. Staroselksy and L. Anand. A constitutive model for hcp materials deforming by slip and twinning: application to magnesium alloy az31b. International Journal of Plasticity, 19:18431864, 2003.

[21] R. A. Lebensohn and C. N. Tomé. A self-consistent viscoplastic model: prediction of rolling textures of anisotropic polycrystals. Mater. Sci. Eng., A175:71-82, 1994.

[22] Sergey Myagchilov and Paul R Dawson. Evolution of texture in aggregates of crystals exhibiting both slip and twinning. Modelling Simul. Mater. Sci. Eng., 7:975-1004, 1999.

[23] Nathan R. Barton, Joel V. Bernier, Ricardo A. Lebensohn, and Donald E. Boyce. The use of discrete harmonics in direct multi-scale embedding of polycrystal plasticity. Comput. Methods Appl. Mech. \& Eng., 283(0):224 - 242, 2015.

[24] I. J. Beyerlein and C. N. Tomé. A probabilistic twin nucleation model for hcp polycrystalline metals. Proc. Roy. Soc. Lond. A, 466(2121):2517-2544, 2010.

[25] G. Proust, C. N. Tomé, A. Jain, and S. R. Agnew. Modeling the effect of twinning and detwinning during strain-path changes of magnesium alloy AZ31. Int. J. Plasticity, 25(5):861$880,2009$.

[26] J.D. Eshelby. The determination of the elastic field of an ellipsoidal inclusion, and related problems. Proceedings of the Royal Society, 241:376-396, 1957.

[27] Hamidreza Abdolvand and Mark R. Daymond. Multi-scale modeling and experimental study of twin inception and propagation in hexagonal close-packed materials using a crystal plasticity finite element part-approach i: Average behavior. J. Mech. Phys. Solids, 61(3):783-802, 2013.

[28] Hamidreza Abdolvand, Marta Majkut, Jette Oddershede, Jonathan P. Wright, and Mark R. Daymond. Study of 3-D stress development in parent and twin pairs of a hexagonal close-packed polycrystal: Part II crystal plasticity finite element modeling. Acta Materialia, 93:235-245, 2015 .

[29] Richard Y. Zhang, Mark R. Daymond, and Richard A. Holt. A finite element model of deformation twinning in zirconium. Mater. Sci. Eng., A, 473(1-2):139 - 146, 2008.

[30] J. Kundin, D. Raabe, and H. Emmerich. A phase-field model for incoherent martensitic transformations including plastic accommodation processes in the austenite. Journal of the Mechanics and Physics of Solids, 59(10):2082 - 2102, 2011.

[31] Tae Wook Heo and Long-Qing Chen. Phase-field modeling of displacive phase transformations in elastically anisotropic and inhomogeneous polycrystals. Acta Materialia, 76:68 - 81, 2014. 
[32] Nathan R. Barton, Joel V. Bernier, Jaroslaw Knap, Anne J. Sunwoo, E. Cerreta, and Todd J. Turner. A call to arms for task parallelism in multi-scale materials modeling. Int. J. Num. Meth. Eng., 86(6):744-764, 2011.

[33] A. Arsenlis, N. R. Barton, R. Becker, and R. E. Rudd. Generalized in situ adaptive tabulation for constitutive model evaluation in plasticity. Comput. Methods Appl. Mech. E5 Eng., 196:1-13, 2006.

[34] Joel V. Bernier, Nathan R. Barton, and Jaroslaw Knap. Polycrystal plasticity based predictions of strain localization in metal forming. J. Eng. Mater. Tech., 130(2):021020, 2008.

[35] Nathan R. Barton, Jaroslaw Knap, Athanasios Arsenlis, Richard Becker, Richard D. Hornung, and David R. Jefferson. Embedded polycrystal plasticity and adaptive sampling. Int. J. Plasticity, 24(2):242-266, 2008.

[36] N. Barton, J.V. Bernier, R. Becker, A. Arsenlis, R. Cavallo, J. Marian, M. Rhee, H.-S. Park, B.A. Remington, and R.T. Olsen. A multiscale strength model for extreme loading conditions. Journal of Applied Physics, 109:073501, 2011.

[37] N. R. Barton, D. J. Benson, and R. Becker. Crystal level continuum modelling of phase transformations: the $\alpha \leftrightarrow \epsilon$ transformation in iron. Modelling Simul. Mater. Sci. Eng., 13:707$731,2005$.

[38] Nathan R. Barton and Hans-Rudolf Wenk. Dauphiné twinning in polycrystalline quartz. Modelling Simul. Mater. Sci. Eng., 15:369-394, 2007.

[39] N. R. Barton, J. V. Bernier, and J. K. Edmiston. Bringing together computational and experimental capabilities at the crystal scale. In Shock Compression of Condensed matter 2009, volume 1195 of AIP Conference Proceedings, pages 73-78. AIP, 2009. Associated with an invited presentation at the SCCM09 conference.

[40] Nathan R. Barton, Nicholas W. Winter, and John E. Reaugh. Defect evolution and pore collapse in crystalline energetic materials. Modelling Simul. Mater. Sci. Eng., 17:035003, 2009. Selected for the MSMSE Highlights of 2009 collection.

[41] N. R. Barton, J. V. Bernier, R. Becker, A. Arsenlis, R. Cavallo, J. Marian, M. Rhee, H.-S. Park, B. Remington, and R. T. Olson. A multi-scale strength model for extreme loading conditions. J. Appl. Phys., 109(7):073501, 2011.

[42] Ryan A. Austin and David L. McDowell. A dislocation-based constitutive model for viscoplastic deformation of fcc metals at very high strain rates. Int. J. Plasticity, In Press, Corrected Proof:-, 2010.

[43] John J. Gilman. Micromechanics of flow in solids. Materials science and engineering. McGrawHill, New York, 1969.

[44] B. Devincre, T. Hoc, and L. Kubin. Dislocation Mean Free Paths and Strain Hardening of Crystals. Science, 320(5884):1745-1748, 2008.

[45] D. S. Drumheller. Introduction to wave propagation in nonlinear fluids and solids. Cambridge University Press, 1998. 
[46] P. P. Gillis, J. J. Gilman, and J. W. Taylor. Stress dependences of dislocation velocities. Phil. Mag., 20(164):279, 1969.

[47] G. I. Taylor. The mechanism of plastic deformation of crystals. Part I. Proc. Roy. Soc. Lond. A, 145:362387, 1934.

[48] Frank J. Zerilli and Ronald W. Armstrong. Dislocation-mechanics-based constitutive relations for material dynamics calculations. Journal of Applied Physics, 61(5):1816-1825, 1987.

[49] H. Mecking and U. F. Kocks. Kinetics of flow and strain-hardening. Acta Metall., 29:1865$1875,1981$.

[50] Matthew Miller and Paul Dawson. Influence of slip system hardening assumptions on modeling stress dependence of work hardening. J. Mech. Phys. Solids, 45(11/12):1781-1804, 1997.

[51] Nathan Barton, Athanasios Arsenlis, Moono Rhee, Jaime Marian, Joel V. Bernier, Meijie Tang, and Lin Yang. A multi-scale strength model with phase transformation. AIP Conference Proceedings, 1426(1):1513-1516, 2012.

[52] Haidong Fan, Sylvie Aubry, Athanasios Arsenlis, and Jaafar A. El-Awady. The role of twinning deformation on the hardening response of polycrystalline magnesium from discrete dislocation dynamics simulations. Acta Materialia, 92:126 - 139, 2015.

[53] Edward M. Breinan and George S. Ansell. The influence of austenite strength upon the austenite-martensite transformation in alloy steels. Met. Trans., 1:1513, June 1970.

[54] A.R. Srinivasa, K.R. Rajagopal, and R.W. Armstrong. A phenomenological model of twinning based on dual reference structures. Acta Materialia, 46(4):1235 - 1248, 1998.

[55] P. Thamburaja, H. Pan, and F.S. Chau. The evolution of microstructure during twinning: Constitutive equations, finite-element simulations and experimental verification. Int. J. Plasticity, 25(11):2141-2168, 2009.

[56] Y. Huo and I. Müller. Nonequilibrium thermodynamics of pseudoelasticity. Continuum Mech. Thermodyn., 5(3):163-204, 1993.

[57] M. Hiratani and E. M. Nadgorny. Combined model of dislocation motion with thermally activated and drag-dependent stages. Acta Mater., 49(20):4337-4346, 2001.

[58] F. Garofalo. Fundamentals of Creep and Creep-Rupture in Metals. MacMillan, New York, NY, 1965 .

[59] Rohan Abeyaratne and James K. Knowles. A continuum model for a thermoelastic solid capable of undergoing phase transitions. J. Mech. Phys. Solids, 41(3):541-571, 1993.

[60] P. S. Follansbee and U. F. Kocks. A constitutive description of the deformation of copper based on the use of the mechanical threshold stress as an internal state variable. Acta Metall., 36(1):81-93, 1988.

[61] M. Kothari and L. Anand. Elasto-viscoplastic constitutive equations for polycrystalline metals: Application to tantalum. J. Mech. Phys. Solids, 46(1):51-67, 1998. 
[62] A. Anderson, R. Cooper, R. Neely, A. Nichols, R. Sharp, and B. Wallin. Users manual for ALE3D - an arbitrary Lagrange/Eulerian 3D code system. Technical Report UCRL-MA152204, Lawrence Livermore National Laboratory, 2003.

[63] E. Orowon and M. Cohen (editor). Dislocations in Metals. Institute of Metals Division, the American Institute of Mining and Metallurgical Engineers, 1954.

[64] B. Clausen, C.N. Tomé, D.W. Brown, and S.R. Agnew. Reorientation and stress relaxation due to twinning: Modeling and experimental characterization for Mg. Acta Mater., 56(11):2456 2468, 2008.

[65] C. Aydıner, Joel Vincent Bernier, Bjørn Clausen, U Lienert, C. Tomé, and D. Brown. Evolution of stress in individual grains and twins in a magnesium alloy aggregate. Physical Review B, 80(2):024113(6), 2009.

[66] S. F. Li and R. M. Suter. Adaptive reconstruction method for three-dimensional orientation imaging. Journal of Applied Crystallography, 46(2):512-524, March 2013.

[67] Joel Vincent Bernier, Nathan Rhodes Barton, Ulrich Lienert, and Matthew Peter Miller. Farfield high-energy diffraction microscopy: a tool for intergranular orientation and strain analysis. The Journal of Strain Analysis for Engineering Design, 46(7):527-547, 2011.

[68] U Lienert, S. F. Li, C. M. Hefferan, J. Lind, R. M. Suter, J. V. Bernier, N. R. Barton, M. C. Brandes, M. J. Mills, M. P. Miller, B. Jakobsen, and W. Pantleon. High-energy diffraction microscopy at the advanced photon source. JOM, 63(7):70-77, 2011.

[69] Jay C Schuren, Paul a Shade, Joel V Bernier, Shiu Fai, Basil Blank, Jonathan Lind, Peter Kenesei, Ulrich Lienert, Robert M Suter, Todd J Turner, Dennis M Dimiduk, and Jonathan Almer. New opportunities for quantitative tracking of polycrystal responses in three dimensions. CURRENT OPINION IN SOLID STATE \& MATERIAL SCIENCE, 2014.

[70] J. Lind, S. F. Li, R. Pokharel, U. Lienert, a. D. Rollett, and R. M. Suter. Tensile twin nucleation events coupled to neighboring slip observed in three dimensions. Acta Materialia, $76: 213-220,2014$.

[71] L. Wang, J. Lind, H. Phukan, P. Kenesei, J.-S. Park, R.M. Suter, A.J. Beaudoin, and T.R. Bieler. Mechanical twinning and detwinning in pure Ti during loading and unloading An in situ high-energy X-ray diffraction microscopy study. Scripta Materialia, 92(October 2015):3538, December 2014.

[72] Hamidreza Abdolvand, Marta Majkut, Jette Oddershede, Jonathan P. Wright, and Mark R. Daymond. Study of 3-D stress development in parent and twin pairs of a hexagonal close-packed polycrystal: Part I in-situ three-dimensional synchrotron X-ray diffraction measurement. Acta Materialia, 93:246-255, 2015.

[73] Jette Oddershede, Søren Schmidt, Henning Friis Poulsen, Henning Osholm Sørensen, Jonathan Wright, and Walter Reimers. Determining grain resolved stresses in polycrystalline materials using three-dimensional X-ray diffraction. Journal of Applied Crystallography, 43(3):539-549, 2010 .

[74] C. Aydner, Joel Vincent Bernier, B Clausen, U Lienert, C. Tomé, and D. Brown. Evolution of stress in individual grains and twins in a magnesium alloy aggregate. Physical Review B, 80(2):024113(6), 2009. 
[75] J.H. Lee, J. Almer, C. Aydıner, Joel Vincent Bernier, K. Chapman, P. Chupas, D. Haeffner, K. Kump, P.L. Lee, U. Lienert, A. Miceli, and G. Vera. Characterization and application of a GE amorphous silicon flat panel detector in a synchrotron light source. Nuclear Instruments and Methods in Physics Research Section A: Accelerators, Spectrometers, Detectors and Associated Equipment, 582(1):182-184, November 2007.

[76] Joel Vincent Bernier. A fast, scalable, and robust orientation indexing algorithm. 2015. in preparation.

[77] Jing Zhang and Shailendra P. Joshi. Phenomenological crystal plasticity modeling and detailed micromechanical investigations of pure magnesium. J. Mech. Phys. Solids, 60(5):945 - 972, 2012 .

[78] C. N. Tomé, S. R. Agnew, W. R. Blumenthal, M. A. M. Bourke, D. W. Brown, G. C. Kaschner, and P. Rangaswamy. The relation between texture, twinning and mechanical properties in hexagonal aggregates. Materials Science Forum, 408:263-268, 2002.

[79] G. Proust, C. N. Tomé, and G. C. Kaschner. Modeling texture, twinning and hardening evolution during deformation of hexagonal materials. Acta Mater., 55(6):2137-2148, 2007.

[80] Haitham El Kadiri, J. Kapil, A.L. Oppedal, L.G. Hector Jr., Sean R. Agnew, M. Cherkaoui, and S.C. Vogel. The effect of twintwin interactions on the nucleation and propagation of twinning in magnesium. Acta Mater., 61(10):3549 - 3563, 2013.

[81] G. T. Gray III, V. Livescu, E. K. Cerreta, T. A. Mason, P. J. Maudlin, and J. F. Bingert. Influence of shockwave obliquity on deformation twin formation in Ta. In DYMAT 2009, pages 963-969, 2009.

[82] J. L. Ericksen. Some surface defects in unstressed thermoelastic solids. Arch. Rational Mech. Anal., 88(4):337-345, 1985.

[83] Malek Homayonifar and Jorn Mosler. Efficient modeling of microstructure evolution in magnesium by energy minimization. International Journal of Plasticity, 28:1-20, 2012.

[84] Dean L. Preston, Davis L. Tonks, and Duane C. Wallace. Model of plastic deformation for extreme loading conditions. J. Appl. Phys., 93(1):211-220, 2003.

[85] William F. Hosford. The Mechanics of Crystals and Textured Polycrystals. Oxford University Press, 1993.

[86] Shuh Rong Chen and George T. Gray III. Constitutive behavior of tantalum and tantalumtungsten alloys. Metall. Mater. Trans. A, 27(10):2994-3006, 1996.

[87] Daniel J. Steinberg. Equation of state and strength properties of selected materials. Technical Report UCRL-MA-106439, Lawrence Livermore National Laboratory, 1996. 


\section{Appendix: Twin plane analysis}

This appendix shows the method used by Ericksen [82] to give analytical results for the two solutions to the twin plane equations. It is based on material presented in [83, 39]. The Hadamard jump condition is a consequence of the continuity of material lines at an interface. The difference in the deformation gradients on either side of the interface is a rank-one tensor. It can be written as

$$
[\mathbf{F}]=\mathbf{a} \otimes \mathbf{N}
$$

where the notation $[\mathbf{F}] \equiv \mathbf{F}_{+}-\mathbf{F}_{-}, \mathbf{a}, \mathbf{N}$ are vectors, $\mathbf{N}$ being the normal to the plane of discontinuity in the reference configuration. This can be rearranged to give

$$
\mathbf{F}_{+}=\left(\mathbf{I}+\mathbf{a} \otimes \mathbf{F}_{-}^{-\mathrm{T}} \mathbf{N}\right) \mathbf{F}_{-}=(\mathbf{I}+\mathbf{b} \otimes \mathbf{n}) \mathbf{F}_{-}
$$

where $\mathbf{b} \equiv\left|\mathbf{F}_{-}^{-\mathrm{T}} \mathbf{N}\right| \mathbf{a}$, and $\mathbf{n}=\frac{\mathbf{F}_{-}^{-\mathrm{T}} \mathbf{N}}{\left|\mathbf{F}_{-}^{-\mathrm{T}} \mathbf{N}\right|}$ is the normal to the surface of discontinuity in the deformed configuration.

Next we form

$$
\mathbf{K} \equiv\left(\mathbf{F}_{+} \mathbf{F}_{-}^{-1}\right)\left(\mathbf{F}_{+} \mathbf{F}_{-}^{-1}\right)^{\mathrm{T}}=(\mathbf{I}+\mathbf{b} \otimes \mathbf{n})(\mathbf{I}+\mathbf{n} \otimes \mathbf{b}),
$$

where we have used Equation 23. Note that $\operatorname{det} \mathbf{K}=1$ for the volume preserving type of twinning considered. This condition implies that $\mathbf{n} \cdot \mathbf{b}=0$, so that the quantity $\mathbf{I}+\mathbf{b} \otimes \mathbf{n}$ has the kinematic interpretation of a simple shear. Since $\mathbf{K}=\mathbf{B B}^{\mathrm{T}}$ is symmetric where $\mathbf{B} \equiv\left(\mathbf{F}_{+} \mathbf{F}_{-}^{-1}\right)$, it has a spectral representation

$$
\mathbf{K}=\lambda_{1} \mathbf{e} \otimes \mathbf{e}+\lambda_{2} \mathbf{f} \otimes \mathbf{f}+\lambda_{3} \mathbf{g} \otimes \mathbf{g},
$$

where $\mathbf{e}, \mathbf{f}$, and $\mathbf{g}$ are the unspecified orthonormal eigenvectors of $\mathbf{K}$, and $\lambda_{i}, i=1,2,3$ are the corresponding principal values. From Equation 24, we see that the action of $\mathbf{K}$ on the vector $\mathbf{h} \equiv \mathbf{b} /|\mathbf{b}| \times \mathbf{n}$ gives

$$
\mathbf{K h}=\mathbf{h},
$$

so that $\mathbf{h}$ is an eigenvector of $\mathbf{K}$ with an associated eigenvalue of 1 . Using this result and noting $\operatorname{det} \mathbf{K}=\lambda_{1} \lambda_{2} \lambda_{3}=1$, the spectral representation simplifies to

$$
\mathbf{K}=\mathbf{h} \otimes \mathbf{h}+\lambda \mathbf{f} \otimes \mathbf{f}+\frac{1}{\lambda} \mathbf{g} \otimes \mathbf{g}
$$

We now work between Equations 24 and 27 to achieve analytical restrictions on the orientation of the twin plan normal $\mathbf{n}$.

Using the representation for $\mathbf{K}$ given in Equation 24, its action on $\mathbf{n}$ is computed as

$$
\mathbf{K n}=\mathbf{n}+\mathbf{b}
$$

where we have used $\mathbf{n} \cdot \mathbf{b}=0$. Next, taking the inner product of Equation 28 with $\mathbf{n}$ and noting $\mathbf{n} \cdot \mathbf{n}=1$ we obtain

$$
\mathbf{K n} \cdot \mathbf{n}=1 .
$$

On the other hand, we apply the same operations to $\mathbf{K}$ using the representation given in Equation 27. Using the result from Equation 29 we get the equation

$$
\mathbf{K n} \cdot \mathbf{n}=\lambda(\mathbf{f} \cdot \mathbf{n})^{2}+\frac{1}{\lambda}(\mathbf{g} \cdot \mathbf{n})^{2}=1
$$


Since $\mathbf{h} \equiv \mathbf{b} /|\mathbf{b}| \times \mathbf{n} \Longrightarrow \mathbf{n} \cdot \mathbf{h}=0$, and the eigenvectors $\mathbf{f}, \mathbf{g}$ are orthonormal, we can completely represent $\mathbf{n}$ on the $\mathbf{f}, \mathbf{g}$ basis and write

$$
\begin{aligned}
\mathbf{n} & =(\mathbf{n} \cdot \mathbf{f}) \mathbf{f}+(\mathbf{n} \cdot \mathbf{g}) \mathbf{g} \\
& =\cos \beta \mathbf{f}+\sin \beta \mathbf{g}
\end{aligned}
$$

where the angle $\beta$ is defined relative to $\mathbf{f}$ in the $\{\mathbf{f}, \mathbf{g}\}$ plane. Substituting Equation 31 into Equation 30 gives the equations

$$
\lambda \cos ^{2} \beta+\frac{1}{\lambda}\left(1-\cos ^{2} \beta\right)=1, \quad \lambda\left(1-\sin ^{2} \beta\right)+\frac{1}{\lambda} \sin ^{2} \beta=1 .
$$

Solving these for $\cos \beta, \sin \beta$ gives the possible solutions for the orientation of the twin plane normal as

$$
\cos \beta= \pm \sqrt{\frac{1}{1+\lambda}}, \quad \sin \beta= \pm \sqrt{\frac{\lambda}{1+\lambda}} .
$$

These four possibilities produce two unique solutions for the twin plane normal. See [82] for comments on the redundancy of some of the solutions given by Equation 33 - that is, some are simply reflections of another.

Example application - magnesium. If one assumes that the parent material has deformation

$$
\mathbf{F}_{-}=\mathbf{I}
$$

then the twinned domain $(+)$ is chosen (independent of the form of (Equation 23)) to have

$$
\mathbf{F}_{+}=\mathbf{I}+\gamma \mathbf{s} \otimes \mathbf{m}
$$

with $\mathbf{s}$ and $\mathbf{m}$ being perpendicular unit vectors $(\mathbf{s} \cdot \mathbf{m}=0)$. This is a simple shear deformation with $\gamma$ being the amount of shear, equal to roughly 0.129 for magnesium for example. Then using Equations 34, 35, and 24 to form $\mathbf{K}$, and solving the eigenvalue problem, we find $\lambda=1.138$ for this case. Equation 33 then gives $\beta=\left\{46.8^{\circ}, 133.2^{\circ}\right\}$ with angles measured from the eigenvector $\mathbf{f}$ toward the eigenvector $\mathbf{g}$ in the $\{\mathbf{f}, \mathbf{g}\}$ plane. For this case, this is the same plane as the $\{\mathbf{s}, \mathbf{m}\}$ plane, and $\mathbf{f}$ falls at $43.2^{\circ}$ measured relative to $\mathbf{s}$, from $\mathbf{s}$ to $\mathbf{m}$. Given that $\beta$ is the angle of $\mathbf{n}$ relative to $\mathbf{f}$, we have, all together, that the twin plane normal $\mathbf{n}$ lies at either $90^{\circ}$ or at $176.3^{\circ}$ relative to $\mathbf{s}$ in the $\{\mathbf{s}, \mathbf{m}\}$ plane.

Now in the simulations, the parent and twin material can both undergo additional deformation such that this precise analysis does not hold and is not directly enforced. However, the results do provide a basis for understanding why the calculations produce two habit planes for a given twin variant at nearly right angles one to another. 


\begin{tabular}{|c|c|c|c|c|}
\hline Parameter & Value & Name & Equation & Reference $(\mathrm{s})$ \\
\hline$g_{\circ} b^{3} / \kappa$ & $230.344 \frac{\mathrm{K}}{\mathrm{GPa}}$ & activation energy parameter & 14,15 & {$[61]$} \\
\hline$\tau_{\mathrm{p}}$ & $400 \mathrm{MPa}$ & Peierls stress & 14,15 & [61] \\
\hline$\dot{\gamma}_{\text {wo }}$ & $0.11361 \mu \mathrm{s}^{-1}$ & ref. thermally activated slip rate & 14,15 & [61] \\
\hline$\dot{\gamma}_{\text {ro }}$ & $8.0985 \mu \mathrm{s}^{-1}$ & ref. drag limited slip rate & 14,13 & [84] \\
\hline$D_{\mathrm{r}}$ & $19.512 \mathrm{GPa}$ & reference drag stress & 13 & \\
\hline$\theta_{\mathrm{o}}$ & $300 \mathrm{~K}$ & reference temperature & 13 & \\
\hline$k_{1}$ & 8359.52 & D.D. production constant & 7 & \\
\hline$k_{2 \mathrm{o}}$ & 491.74 & D.D. production constant & 7 & \\
\hline $1 / n$ & 0.25 & D.D. rate parameter & 7 & \\
\hline$\dot{\gamma}_{\mathrm{ko}}$ & $0.001 \mu \mathrm{s}^{-1}$ & D.D. rate parameter & 7 & \\
\hline$\left.h\right|_{t=0}$ & 1 & initial relative D.D. & 7 & \\
\hline$g_{\mathrm{o}}$ & $0 \mathrm{MPa}$ & slip resistance constant & 5 & \\
\hline$s$ & $7.8936 \mathrm{MPa}$ & slip resistance constant & 5 & \\
\hline$c_{11}$ & $260.2 \mathrm{GPa}$ & elastic moduli & & {$[85,61]$} \\
\hline$c_{12}$ & $154.5 \mathrm{GPa}$ & elastic moduli & & {$[85,61]$} \\
\hline$c_{44}$ & $82.6 \mathrm{GPa}$ & elastic moduli & & {$[85,61]$} \\
\hline$c_{\mathrm{v}}$ & $0.1455 \frac{\mathrm{kJ}}{\mathrm{kg} \cdot \mathrm{K}}$ & heat capacity & & [86] \\
\hline $\bar{\rho}$ & $16.6 \frac{\mathrm{g}}{\mathrm{cm}^{3}}$ & reference density & & [86] \\
\hline$S$ & 1.2 & EOS & & [87] \\
\hline$\Gamma_{\mathrm{o}}$ & 1.67 & EOS & & {$[87]$} \\
\hline$a$ & 0.42 & EOS & & {$[87]$} \\
\hline$\xi_{\mathrm{t}}$ & $10 \mathrm{MPa}$ & twin threshold & 8 & \\
\hline$\xi_{\mathrm{o}}$ & $50 \mathrm{MPa}$ & twin resistance constant & 8,9 & \\
\hline$\xi$ & $20 \mathrm{MPa}$ & twin resistance constant & 8,9 & \\
\hline$r$ & 0.1 & twin rate sensitivity & 8 & \\
\hline$\dot{\Phi}^{\mathrm{o}}$ & $100 \mu \mathrm{s}^{-1}$ & reference twinning rate & 8 & \\
\hline$C^{\mathrm{r}_{\mathrm{a}}}$ & $100 \mathrm{MPa}$ & interaction parameter & 11 & \\
\hline$C^{\mathrm{r}_{\mathrm{b}}}$ & $100 \mathrm{MPa}$ & interaction parameter & 11 & \\
\hline
\end{tabular}

Table 1: Table of material parameters used for tantalum. 


\begin{tabular}{|c|c|c|c|c|}
\hline Parameter & Value & Name & Equation & Reference(s) \\
\hline$\dot{\gamma}_{\text {wo }}$ & $1 \cdot 10^{-3} \mu \mathrm{s}^{-1}$ & ref. thermally activated slip rate & 14,15 & [61] \\
\hline$\dot{\gamma}_{\text {ro }}$ & $1 \cdot 10^{3} \mu \mathrm{s}^{-1}$ & ref. drag limited slip rate & 14,13 & [84] \\
\hline$D_{\mathrm{r}}$ & $400 \mathrm{GPa}$ & reference drag stress & 13 & \\
\hline$\theta_{\mathrm{o}}$ & $300 \mathrm{~K}$ & reference temperature & 13 & \\
\hline$k_{1}$ & 102 & D.D. production constant & 7 & \\
\hline$k_{2 \mathrm{o}}$ & 6.9 & D.D. production constant & 7 & \\
\hline $1 / n$ & 0.0 & D.D. rate parameter & 7 & \\
\hline$\left.h\right|_{t=0}$ & 1 & initial relative D.D. & 7 & \\
\hline$g_{\mathrm{o}}$ & $11.970 \mathrm{MPa}$ & slip resistance constant & 5 & \\
\hline$s$ & $7.14 \mathrm{MPa}$ & slip resistance constant & 5 & \\
\hline$c_{11}$ & $58.46 \mathrm{GPa}$ & elastic moduli & & \\
\hline$c_{12}$ & $24.96 \mathrm{GPa}$ & elastic moduli & & \\
\hline$c_{13}$ & $20.82 \mathrm{GPa}$ & elastic moduli & & \\
\hline$c_{33}$ & $61.00 \mathrm{GPa}$ & elastic moduli & & \\
\hline$c_{44}$ & $16.58 \mathrm{GPa}$ & elastic moduli & & \\
\hline$c_{\mathrm{V}}$ & $1.25 \frac{\mathrm{kJ}}{\mathrm{kg} \cdot \mathrm{K}}$ & heat capacity & & \\
\hline $\bar{\rho}$ & $1.78 \frac{\mathrm{g}}{\mathrm{cm}^{3}}$ & reference density & & \\
\hline$S$ & 1.242 & EOS & & [87] \\
\hline$\Gamma_{\mathrm{o}}$ & 1.54 & EOS & & {$[87]$} \\
\hline$a$ & 0.33 & EOS & & [87] \\
\hline$\xi_{\mathrm{t}}$ & $0 \mathrm{MPa}$ & twin threshold & 8 & \\
\hline$\xi_{\mathrm{o}}$ & $3 \mathrm{MPa}$ & twin resistance constant & 8,9 & \\
\hline$\xi$ & $0.651 \mathrm{MPa}$ & twin resistance constant & 8,9 & \\
\hline$r$ & 0.05 & twin rate sensitivity & 8 & \\
\hline$\dot{\Phi}^{\mathrm{o}}$ & $0.001 \mu \mathrm{s}^{-1}$ & reference twinning rate & 8 & \\
\hline$C^{\mathrm{r}_{\mathrm{a}}}$ & $7.27 \mathrm{MPa}$ & interaction parameter & 11 & \\
\hline$C^{\mathrm{rb}_{\mathrm{b}}}$ & $1.21 \mathrm{MPa}$ & interaction parameter & 11 & \\
\hline
\end{tabular}

Table 2: Table of material parameters used for Magnesium AZ31. 


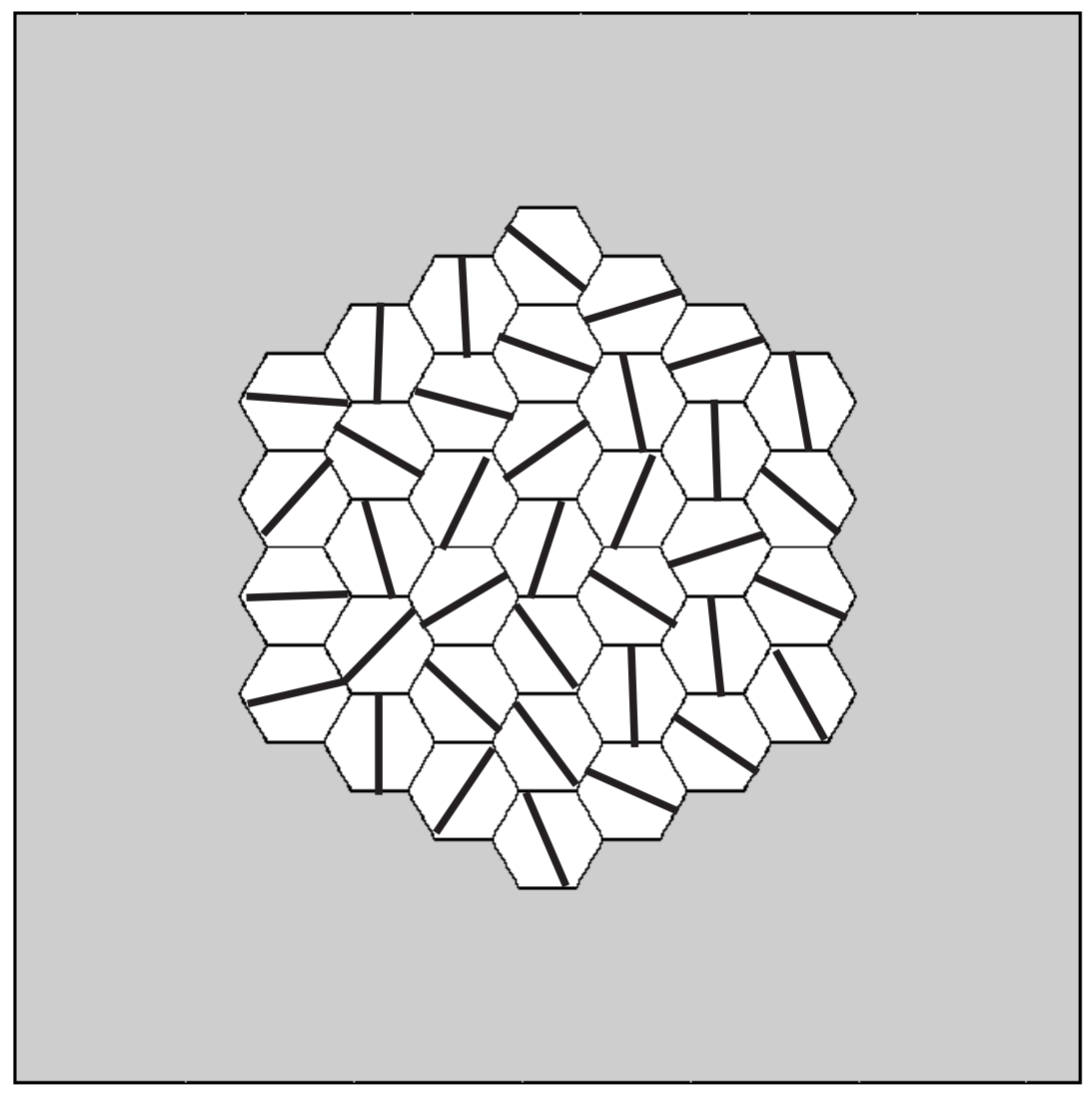

Figure 1: Initial microstructure with 37 hexagonal grains inside a homogeneous elastic medium which is shown in gray. The twin shear direction of the single twin system in each grain is indicated by the thick black line within each grain. 


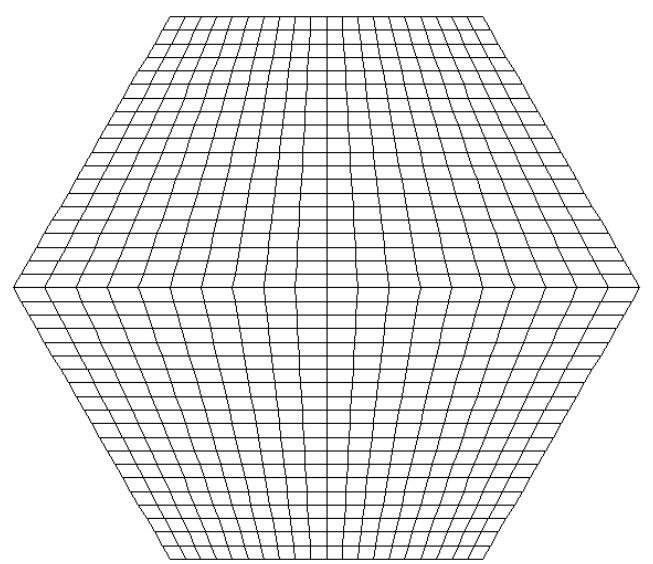

Figure 2: Mesh used for each single grain in the Ta simple shear simulation. 

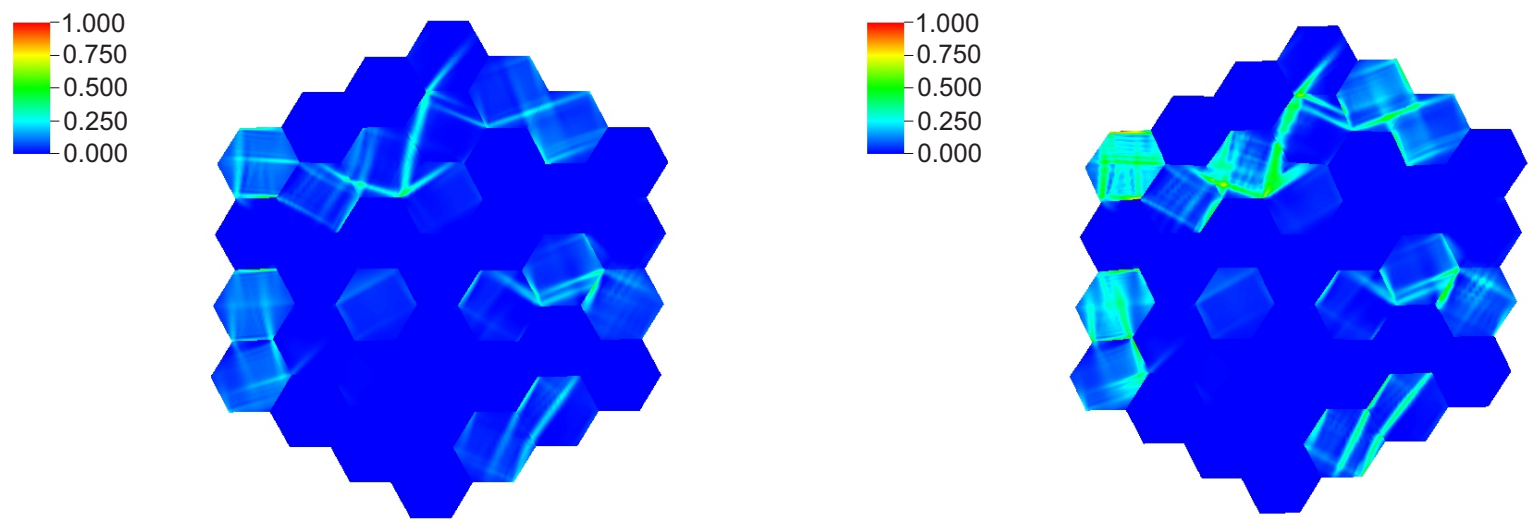

(a)

-1.000
-0.750
-0.500
-0.250
0.000

(b)

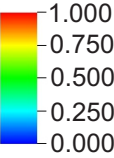

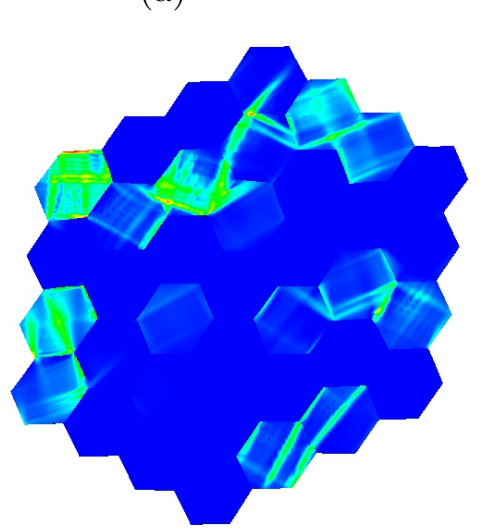

(c)

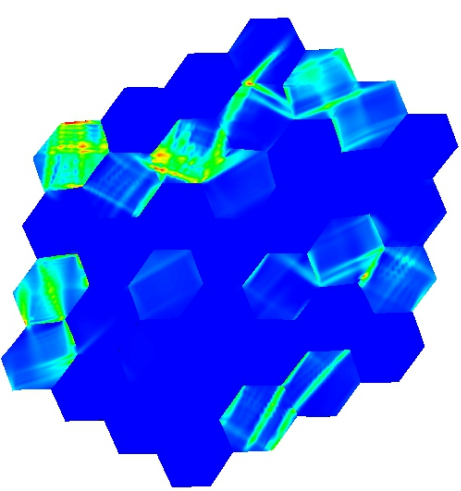

(d)

Figure 3: The twin mass fraction for an imposed strain rate of $10^{5} /$ second at; (a) $\gamma=0.0375$, (b) $\gamma=0.075$, (c) $\gamma=0.1125$, and (d) $\gamma=0.15$. We have not shown the homogeneous elastic medium for the sake of clarity. 

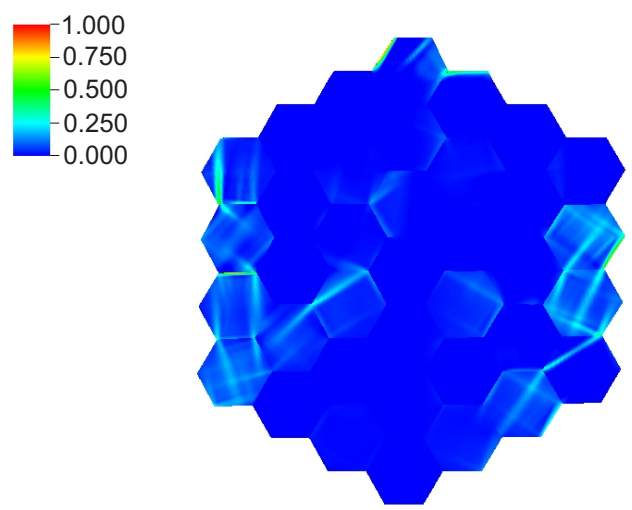

(a)

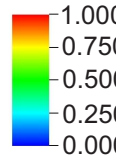

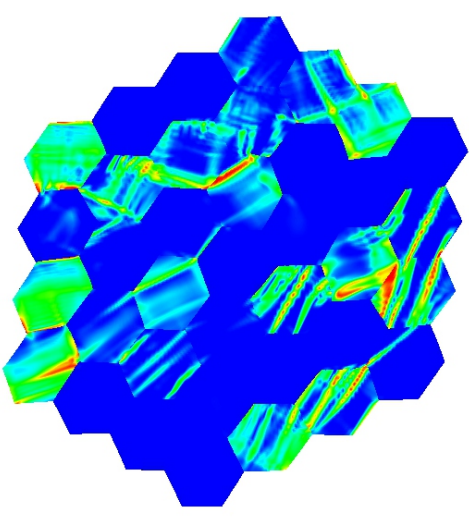

(c)
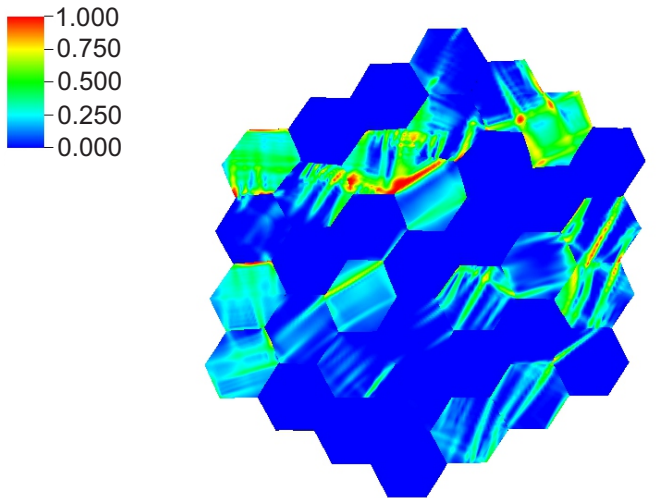

(b)
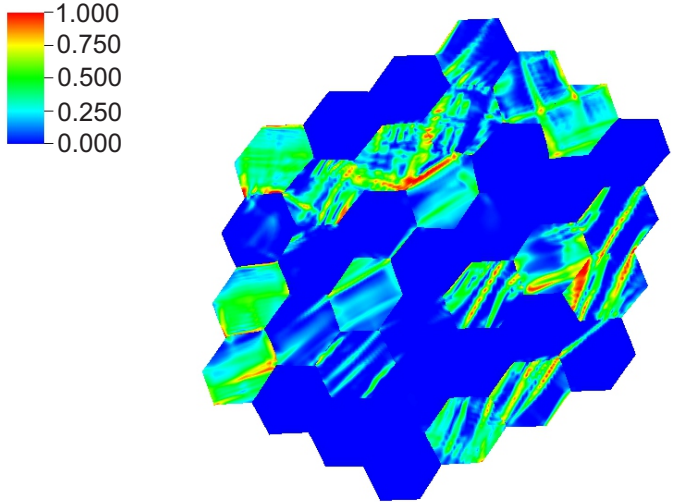

(d)

Figure 4: The twin mass fraction for an imposed strain rate of $10^{6} /$ second at; (a) $\gamma=0.0375$, (b) $\gamma=0.075$, (c) $\gamma=0.1125$, and (d) $\gamma=0.15$. We have not shown the homogeneous elastic medium for the sake of clarity. 


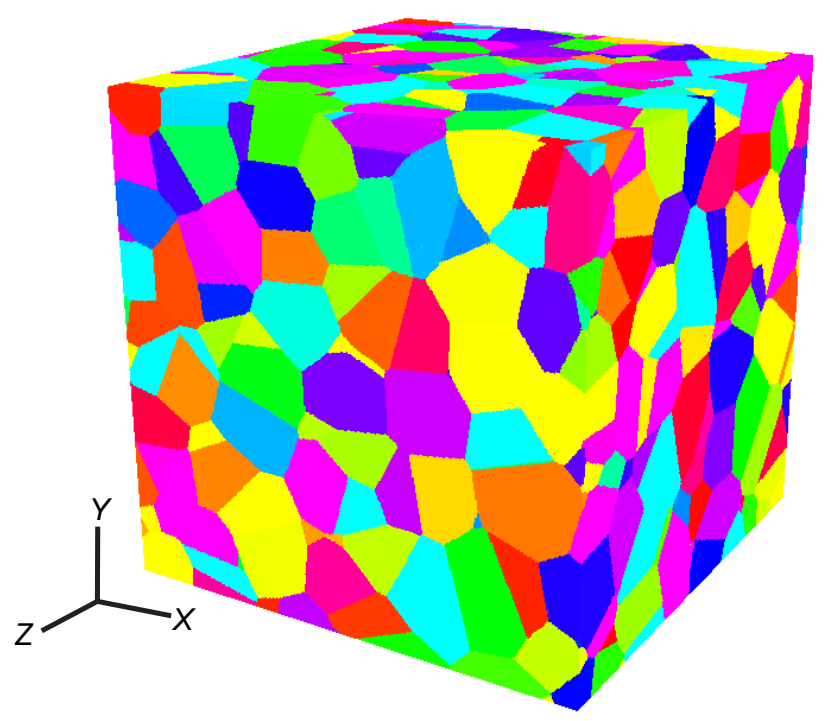

Figure 5: Initial microstructure, consisting of 512 grains in a $60 \times 60 \times 60 \mu \mathrm{m}$ domain, used for the Ta polycrystal compression simulation. It is worth noting that while the colors distinguish the individual grains in the domain, the color mapping does not take into account the periodic nature of this microstructure. Here, the loading is along the y-direction, and the lateral directions (normals along $\mathrm{x}$ - and $\mathrm{z}$-directions) are periodic in crystal orientation. 

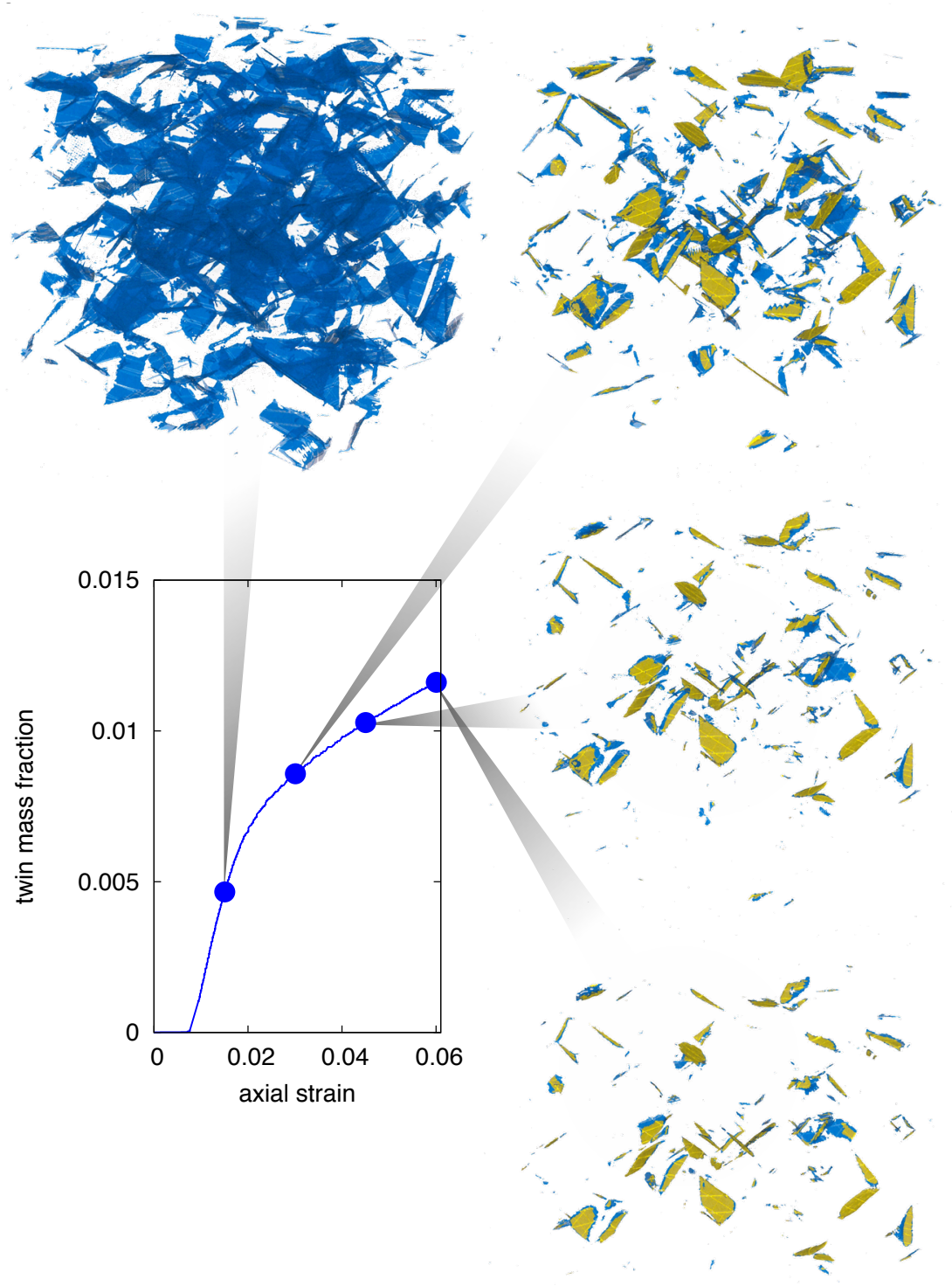

Figure 6: The predicted twin mass fraction evolution for the Ta polycrystal, along with some snapshots showing isosurfaces (drawn at $25 \%$ of the maximum at that time) of the parent mass fraction at axial strains of $0.015,0.03,0.045$, and 0.06 ; with isosurfaces drawn at 0.0178, 0.0704, 0.15 , and 0.25 , respectively, note the scale is not uniform. Here, yellow indicates the twin mass fraction from the previous time, while blue shows the the current time. 


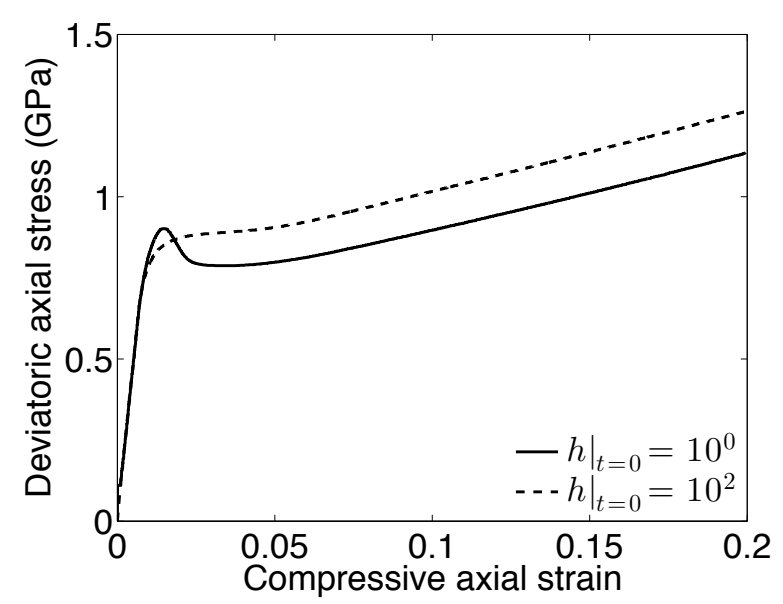

a)

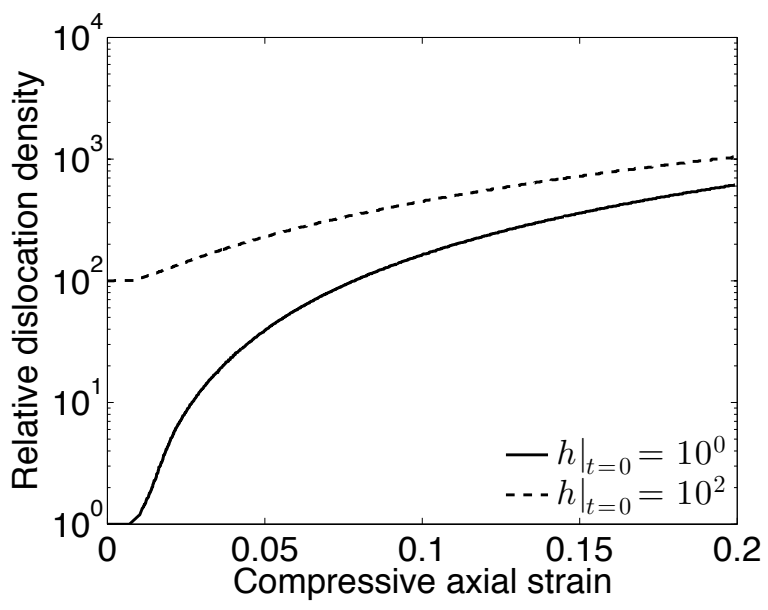

b)

Figure 7: a) The stress-strain response of the Ta polycrystal and b) the corresponding evolution of the dislocation density, for the two cases of initial dislocation density. Here, the reference state corresponds to an initial dislocation density of an annealed sample while the high initial dislocation density is two orders of magnitude higher, to serve as the initial condition from a modest amount of cold working. It is interesting to note that the reference state exhibits twinning, while the high initial dislocation density does not show any twinning. However, once the dislocation density of the reference goes above 100, it still continues to twin. 


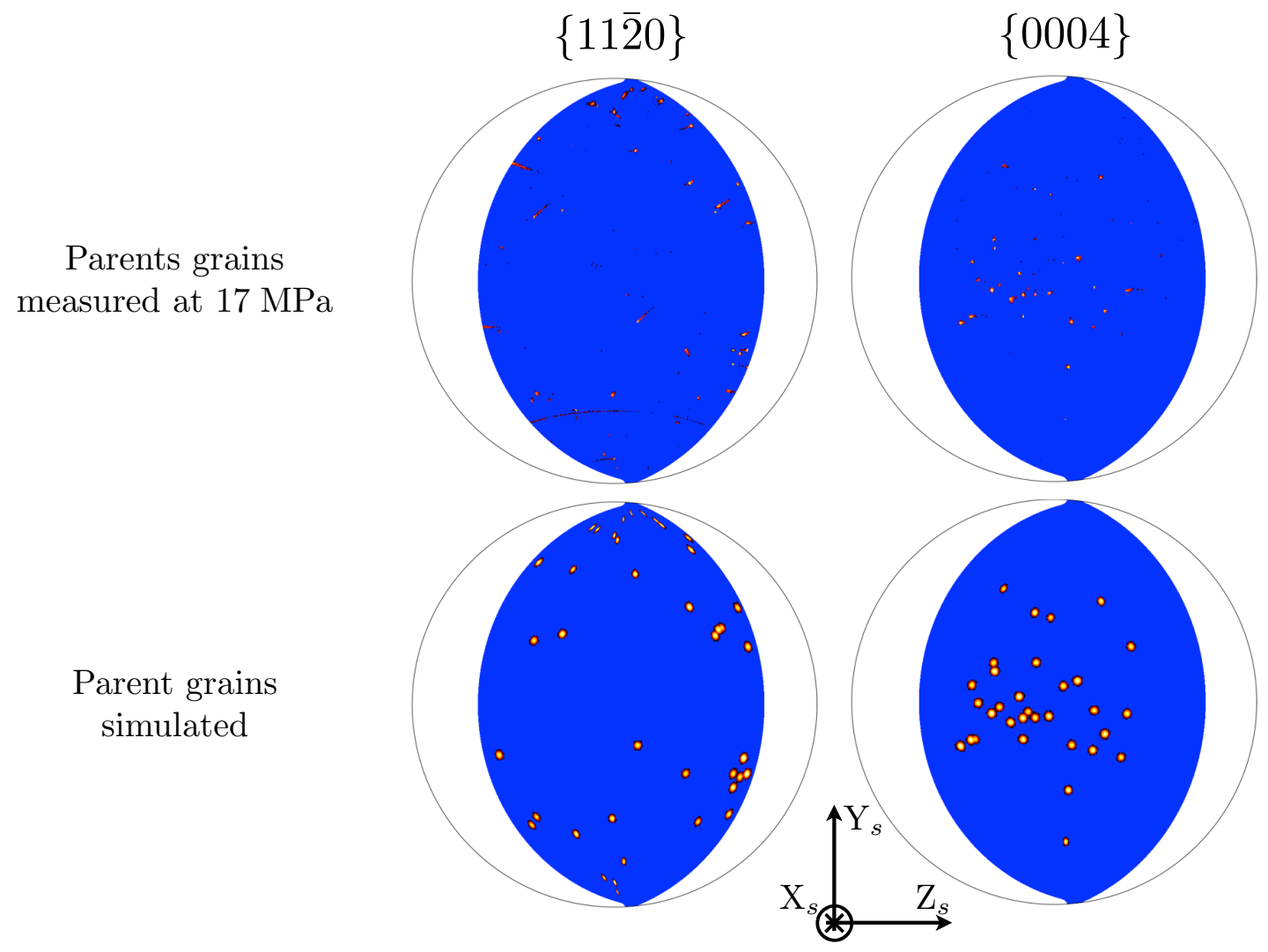

Figure 8: Dicrete pole figures (i.e. $\eta-\omega$ maps) for the initial microstructure. The colormap uses the $\log$ of the raw binned intensities, which increases the visibility of weaker features, and the blue fill denotes the signal to noise threshold. Streaking of the bragg reflections in the measured pole figures is indicative of some initial orientation spread in the grains, which is not represented in the initial mesh for the simulation. The preferred orientation imparted by the processing manifests in the clustering of the basal planes near the equator in the $\{0004\}$ pole figure. The simulated pole figures were constructed using gaussians for each grains' visible reflections with intensities scaled by the volume of each grain (from the voronoi tesselation) in the initial mesh; the standard deviations are exaggerated to aid visualization. 


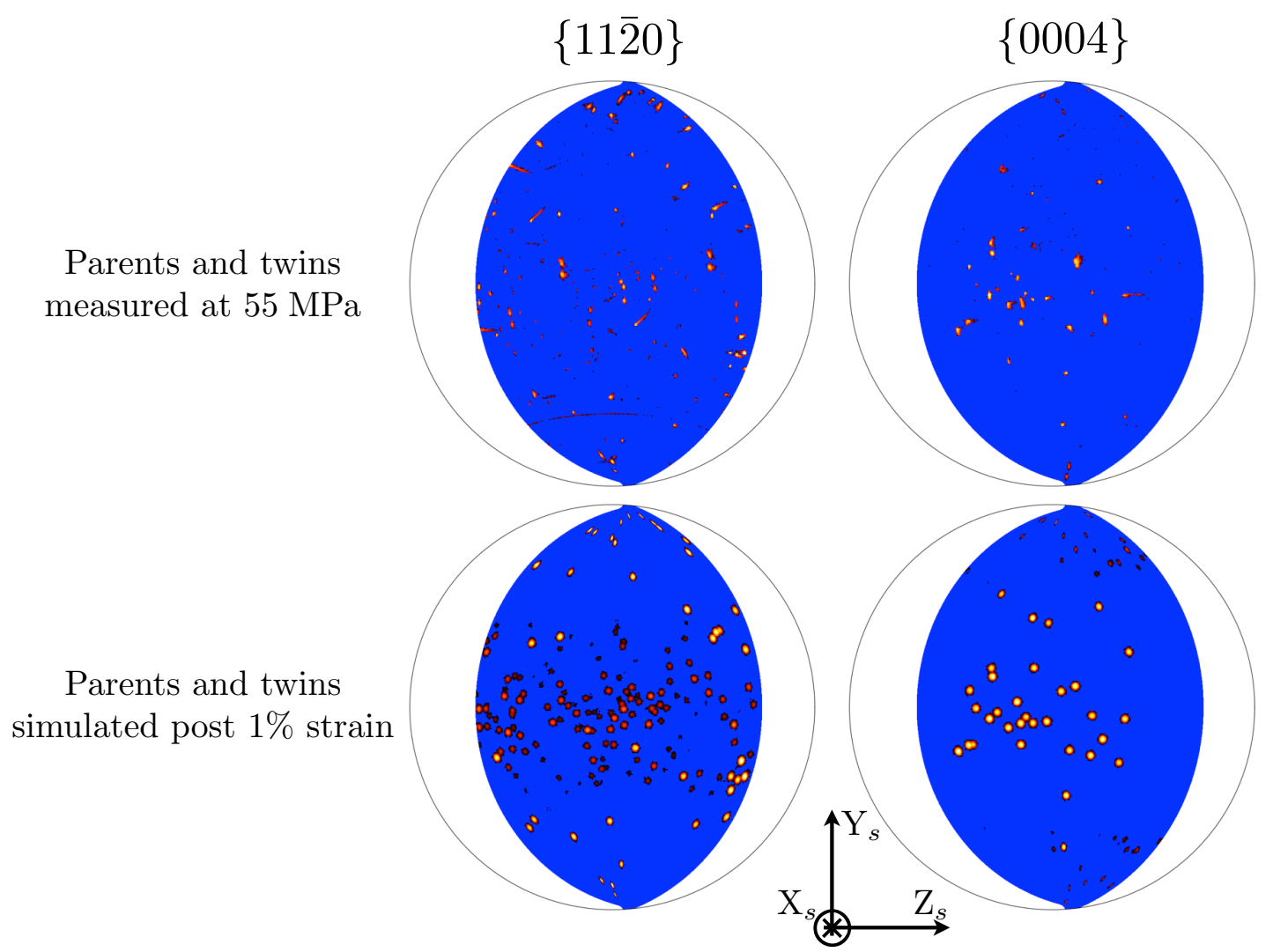

Figure 9: Dicrete pole figures after twinning. In the measurement, twins were clearly visible at the $55 \mathrm{MPa}$ load step and approximately $1 \%$ strain. The simulation results are after $1 \%$ applied strain. Due to the initial texture and the preferred twin variant selection, the signals from the twins appear near the equator of the $\{11 \overline{2} 0\}$ pole figure and near the poles of the $\{0004\}$ pole figure. There is a reasonable degree of similarity between the measured and simulated results. Twin reflection intensities are scaled by the corresponding mass fractions predicted by the simulation. 


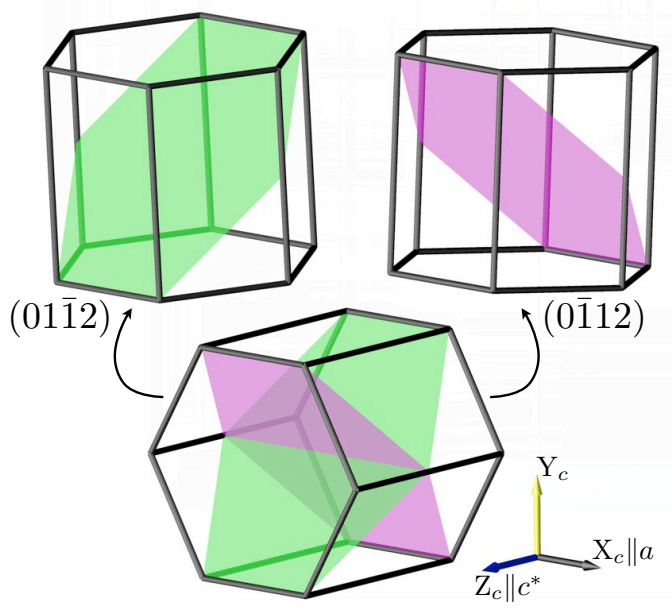

Figure 10: Schematic illustrating the "crossed" tensile twin variant pairs most commonly observed in both the measurement and simulation. Shown here are variants 2 (green) and 5 (magenta) in the chosen numbering convention, where $\{1,2,3\}$ and $\{4,5,6\}$ form sets of opposing planes. The upper hexagonal cells represent the actual lattice orientation relative the the parent (lower cell) with the corresponding $\{10 \overline{1} 2\}$ planes highlighted. The coloring matches that used in Figure 12. 


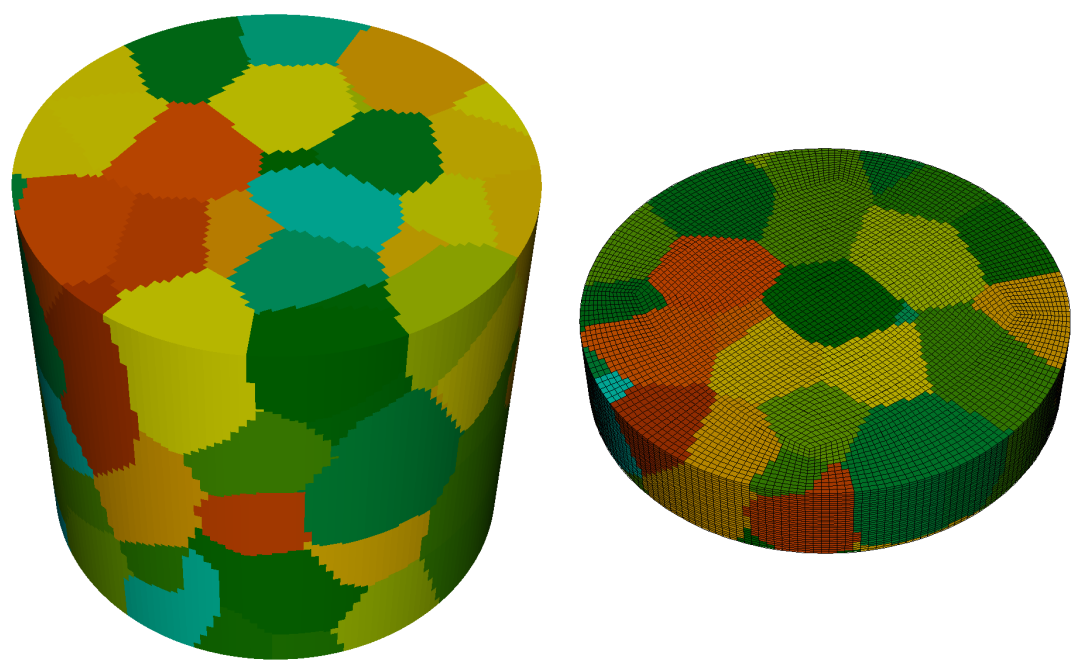

Figure 11: (left) Initial grain structure taken from the experiment and used for the AZ31 compression simulation. (right) The mesh shown indicates the central region where experimental data are compared against the simulation. 


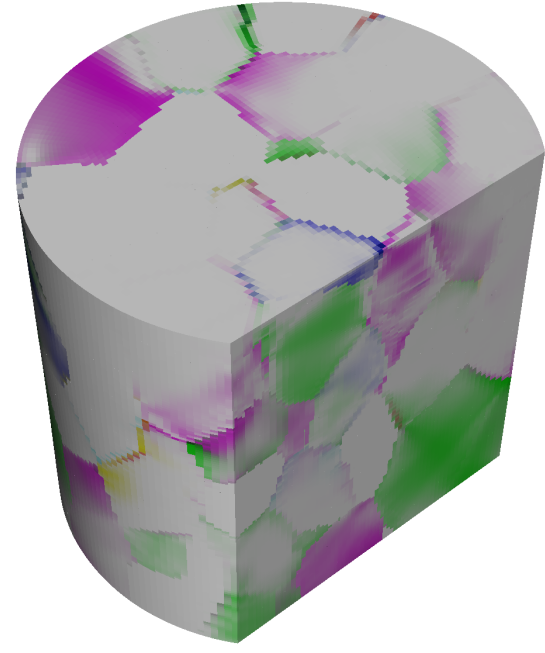

(a) Twin variant mass fractions

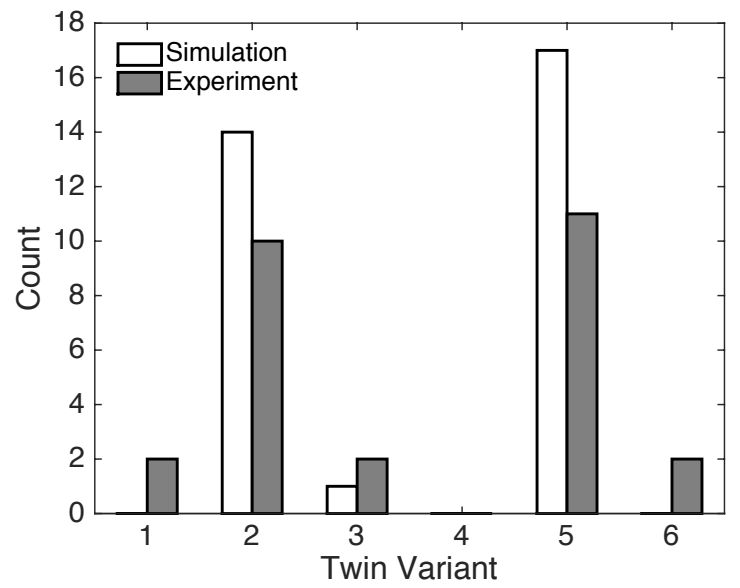

(b) Observed variants by label

Figure 12: Twin variant selection results among the six distinct types. Inset a) shows mass fractions of twins at a strain of roughly $1 \%$ axial compressive strain. Here the colors differentiate which twin variants are present as described in the body. Inset b) show the comparison between the experimental and simulated twin variants in the Mg AZ31 sample. The values are chosen in the experimental measurement when over $50 \%$ complete, and in the simulation when the mass fraction is greater than $1 \%$, which corresponds to a reasonable detection threshold. 


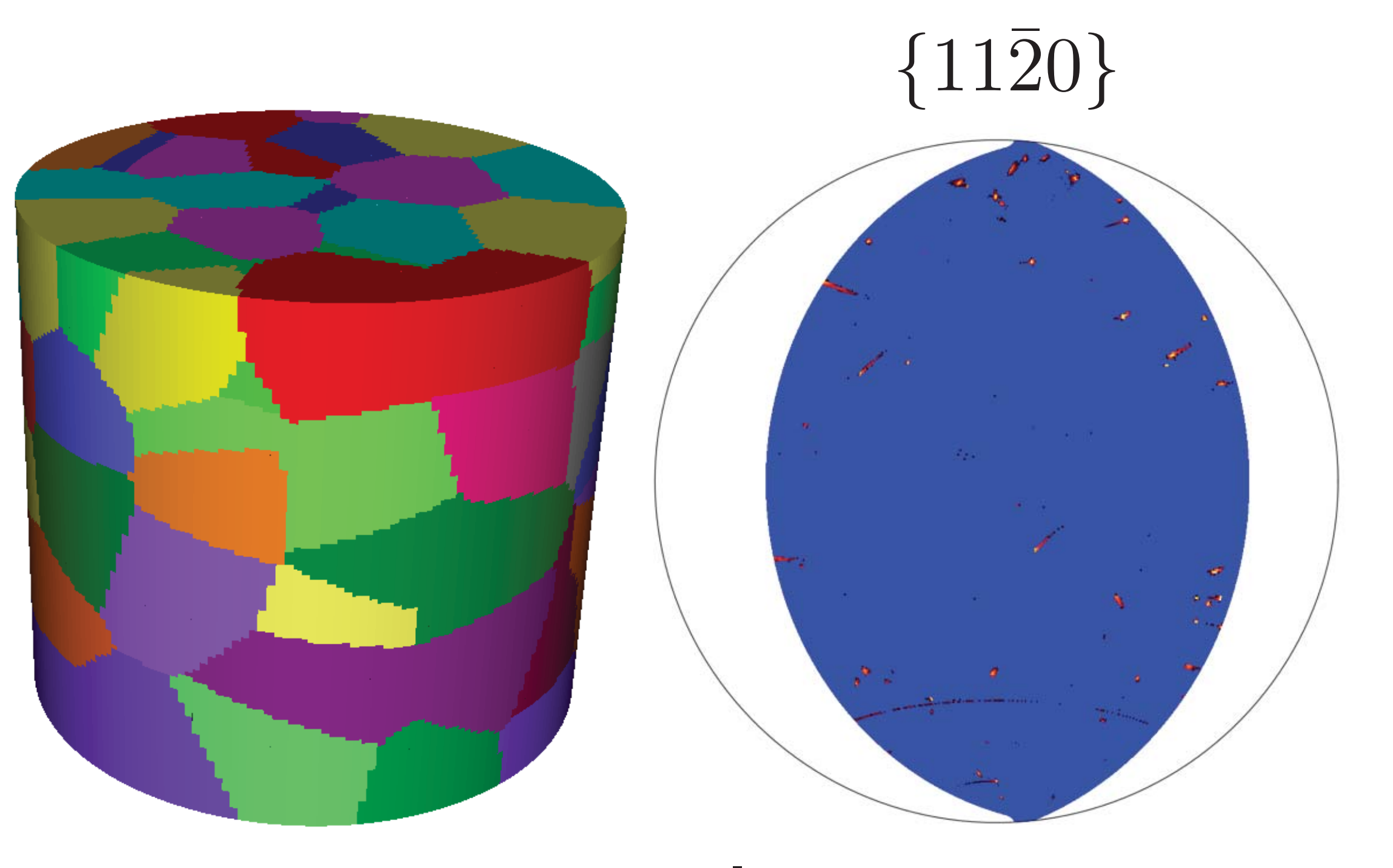

Initial

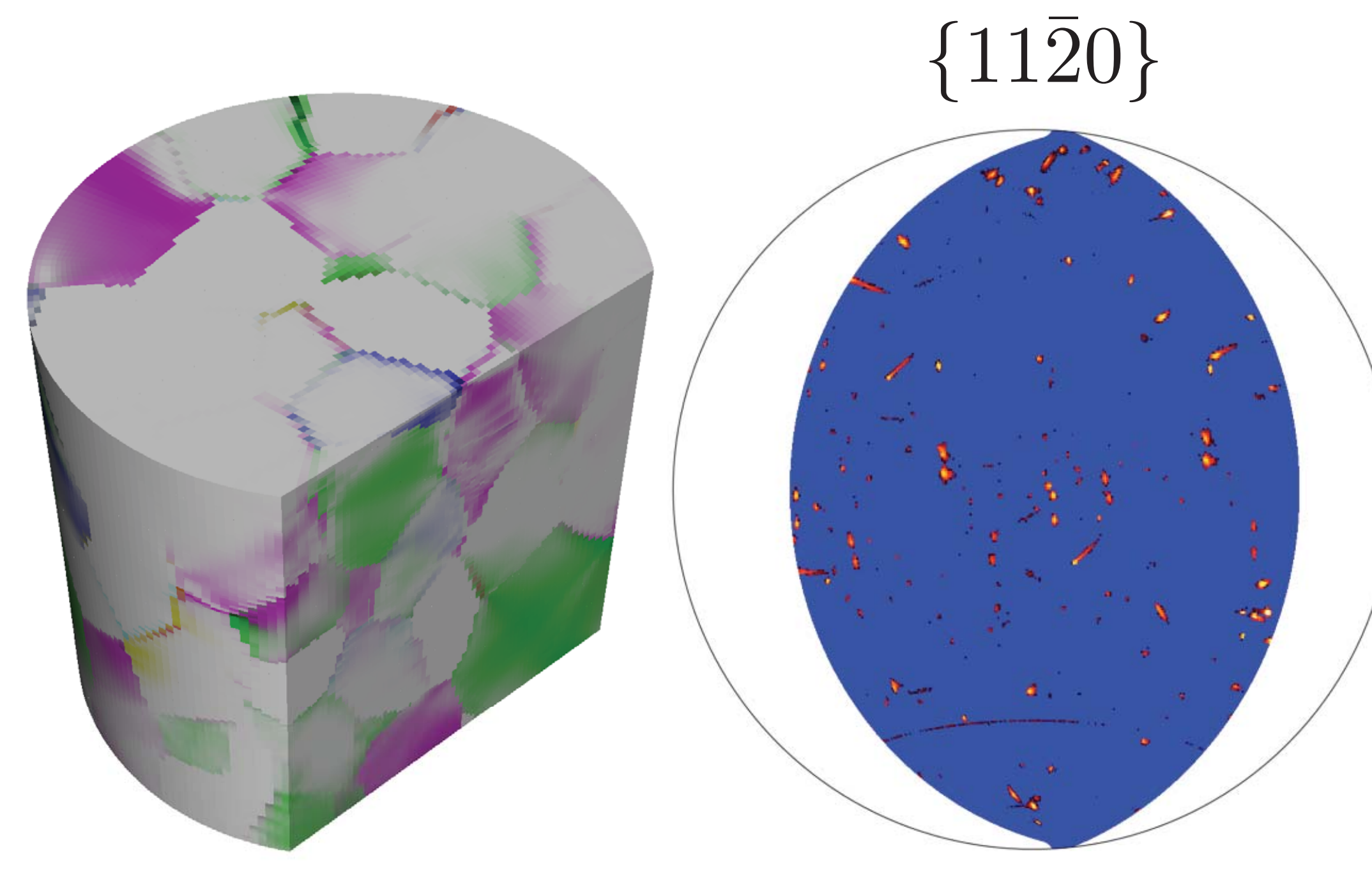

Deformed 